Portland State University

PDXScholar

Spring 6-14-2013

\title{
Automated Channel Assessment for Single Chip MedRadio Transceivers
}

Mark Alexander Hillig

Portland State University

Follow this and additional works at: https://pdxscholar.library.pdx.edu/open_access_etds

Part of the Biomedical Commons, Electronic Devices and Semiconductor Manufacturing Commons, Equipment and Supplies Commons, and the Signal Processing Commons Let us know how access to this document benefits you.

\section{Recommended Citation}

Hillig, Mark Alexander, "Automated Channel Assessment for Single Chip MedRadio Transceivers" (2013). Dissertations and Theses. Paper 1005.

https://doi.org/10.15760/etd.1005

This Thesis is brought to you for free and open access. It has been accepted for inclusion in Dissertations and Theses by an authorized administrator of PDXScholar. Please contact us if we can make this document more accessible: pdxscholar@pdx.edu. 


\author{
by \\ Mark Alexander Hillig
}

A thesis submitted in partial fulfillment of the requirements for the degree of

\author{
Master of Science \\ in \\ Electrical and Computer Engineering
}

Thesis Committee:

Fu Li, Chair

Marek A. Perkowski

James E. Morris

Portland State University
2013 


\begin{abstract}
Modern implantable and body worn medical devices leverage wireless telemetry to improve patient experience and expand therapeutic options. Wireless medical devices are subject to a unique set of regulations in which monitoring of the available frequency spectrum is a requirement. To this end, implants use software protocols to assess the in-band activity to determine which channel should be used. These software protocols take valuable processing time and possibly degrade the operational lifetime of the battery. Implantable medical devices often take advantage of a single chip transceiver as the physical layer for wireless communications. Embedding the channel assessment task in the transceiver hardware would free the limited resources of the microprocessor.

This thesis proposes hardware modifications to existing transceiver architectures which would provide an automated channel assessment means for implantable medical devices. The results are applicable beyond medical device applications and could be employed to benefit any low-power, wireless, battery-operated equipment.
\end{abstract}




\section{Acknowledgements}

I would like to thank Micro Systems Engineering Incorporated for providing me the resources needed to complete this thesis. Special thanks to Brian Sutton and Paul Stadnik for guidance through its development.

I would also like to acknowledge the guidance of $\mathrm{Dr}$. Fu $\mathrm{Li}$ throughout my graduate studies and thesis. Additional thanks to Dr. Marek Perkowski and Dr. James Morris for participating on my thesis committee. 


\section{Table of Contents}

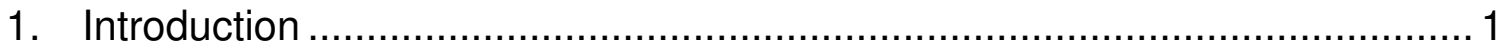

2. Single Chip Radio Technology ........................................................... 3

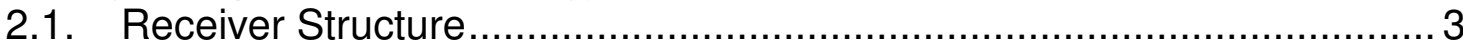

2.2. Power Usage in Single Chip Transceivers ....................................... 5

2.3. Channel Assessment...................................................................... 7

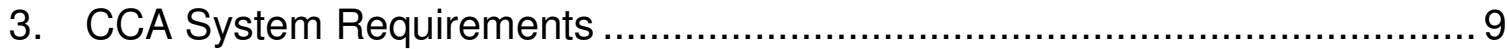

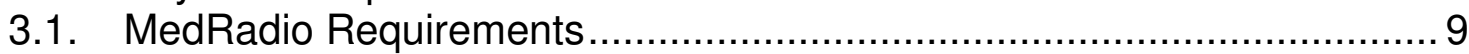

3.2. Channel Assessment Time ............................................................... 10

3.3. Channel Assessment Protocol ..................................................... 12

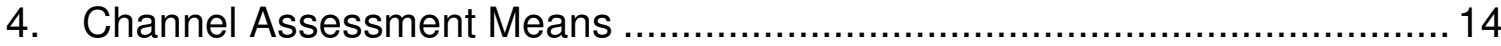

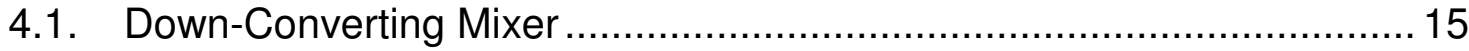

4.2. Frequency Source .................................................................... 16

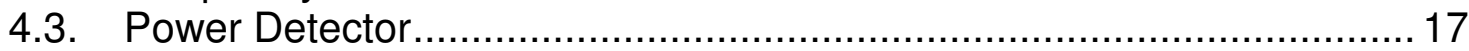

4.4. Control Output and Measurement …............................................. 17

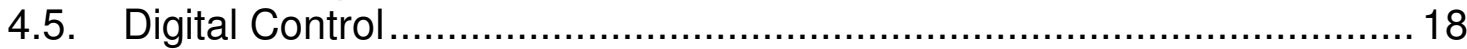

4.6. Hardware Impact on Transceiver Size ............................................2 21

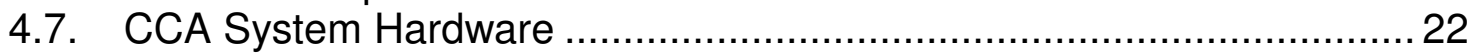

4.8. Hardware Timing Considerations .................................................... 23

4.9. Signal Power Level and Power Consumption Considerations...............24

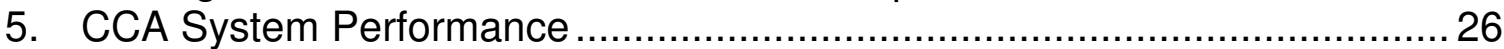

5.1. CCA Test Setup and Conditions.....................................................26

5.2. Timing Confirmation ................................................................... 28

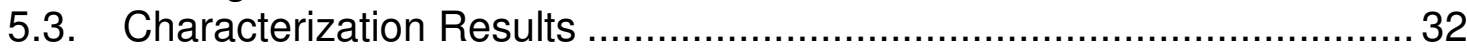

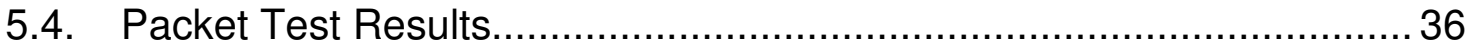

5.5. Threshold Detection Vs. Channel Energy Assessment ......................... 39

6. Possible Improvements to the CCA System ............................................. 41

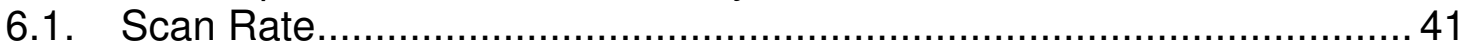

6.2. Mixer Type Selection .................................................................. 41

6.3. Multi-Level Reporting or Automatic Threshold Adjustment.................... 42

6.4. Integration with the Larger Transceiver System ................................ 42

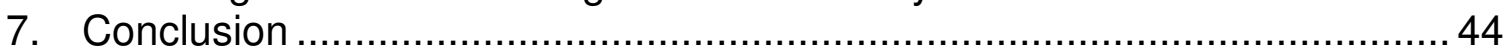

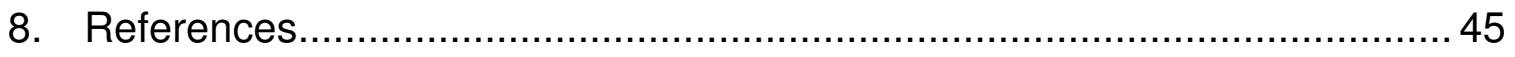

Appendix A. Verilog Source Code for Clear Channel Assessment .....................50

Appendix B. Schematics for RF, DAC, and ADC Hardware .............................54 


\section{Tables}

Table 1 - A comparison of the apparent architecture of 13 commercial chip transceivers.

Table 2 - The stated current consumption for various operating modes for each of

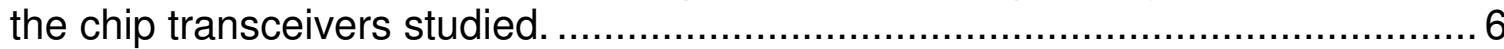

Table 3 - CCA packet test performance with 34-channels............................... 38

Table 4 - CCA packet test performance with 12-channels................................ 39 


\section{Figures}

Figure 1 -The direct-conversion reciever architecture. .................................... 4

Figure 2 - Limiter amplifier chain for receiver gain control. ................................ 5

Figure 3 - Hypothetical, three-part, bidirectional protocol for a two-node

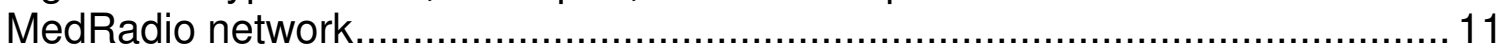

Figure 4 - Potential measurement points within a channel. ............................. 13

Figure 5 - An unsuppressed image signal from a DSB mixer appearing in the IF

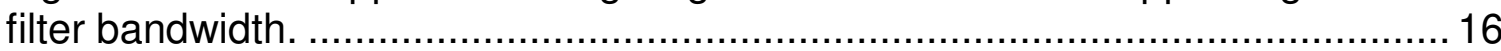

Figure 6 - Block diagram of the study system ............................................... 18

Figure 7 - Block diagram of the digital control hardware.................................2 20

Figure 8 - State flow diagram of the hardware finite state machine (FSM) ........21

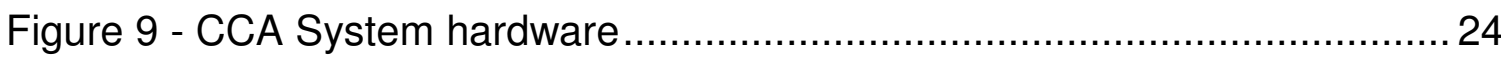

Figure 10 - Test setup used to evaluate the proposed CCA system................... 27

Figure 11 - SMIQ controller software written in C\# for packet testing................. 28

Figure 12 - Accumulated spectrum for the VCO output with 12 channels. .........29

Figure 13 - Accumulated spectrum for the VCO output with 34 channels. ......... 30

Figure 14 - DAC/ADC write/read timing and VCO output using 34 channels. .... 31

Figure 15 - DAC/ADC write/read timing and VCO output using 34 channels. .... 32

Figure 16 - The WCDMA test signal and the CCA system. ............................. 34

Figure 17 - The Bluetooth modulated test signal and the CCA system output. .. 35

Figure 18 - The 2FSK test signal and the CCA system output. ........................ 36 


\section{Equations}

Equation 1 - Detection threshold of occupied channels in the $402-405 \mathrm{MHz}$ band. 10

Equation 2 - Carson's bandwidth rule and the resulting detection threshold. ..... 10 


\section{Acronyms and Abbreviations}

$\begin{array}{ll}\text { ADC } & \text { analog to digital converter } \\ \text { ASK } & \text { amplitude shift key } \\ \text { CCA } & \text { clear channel assessment } \\ \text { CMOS } & \text { complementary metal oxide semiconductor } \\ \text { DAC } & \text { digital to analog converter } \\ \text { DSB } & \text { double-sideband mixer } \\ \text { FPGA } & \text { field programmable gate array } \\ \text { FSK } & \text { frequency shift key } \\ \text { IF } & \text { intermediate frequency } \\ \text { kbps } & \text { kilobits per second } \\ \text { kHz } & \text { kilohertz } \\ \text { LBT } & \text { listen-before-talk } \\ \text { LNA } & \text { low noise amplifier } \\ \text { LO } & \text { local oscillator } \\ \text { mA } & \text { milliamperes } \\ \text { MedRadio } & \text { 47 CFR 95.627; 47 CFR 95.628 } \\ \text { MHz } & \text { megahertz } \\ \text { MICS } & \text { Medical Implant Communications Service } \\ \text { mS } & \text { milliseconds } \\ \text { PLL } & \text { phase-lock-loop } \\ \text { RF } & \text { radio frequency } \\ \text { RSSI } & \text { received signal strength indicator } \\ \text { RX } & \text { receive } \\ \text { SSB } & \text { single side-band } \\ \text { UART } & \text { universal asynchronous receiver/transmitter } \\ \mu S & \text { microseconds } \\ \text { VCO } & \text { voltage controlled oscillator } \\ \text { VGA } & \text { variable gain amplifier } \\ \text { WCDMA } & \text { wide band code division multiple access } \\ & \end{array}$


1. Introduction

Implantable and body worn medical devices are available for a wide range of applications such as pacemakers, insulin pumps, and neurological stimulation. These devices significantly benefit from wireless connectivity, aiding both the physician and the patient by allowing communication with the device without the need for physical contact or a medical office visit. With an increase in use of wireless connectivity, the limited MedRadio frequency spectrum [1], [2] is becoming crowded. For low-power, battery-operated, wireless nodes such as medical devices, crowded channels can directly impact the operational lifetime of the network [3]. As a result, clear channel assessment (CCA) techniques [4] are of critical importance to maintaining wireless link integrity while reducing radio on-time.

A hardware mechanism that gives insight into channel activity could provide lowpower networks a means to maintain timely access without compromising operational lifetime. Significant research has been done in the design and study of MedRadio transceivers [5]-[10] however, none of these efforts have demonstrated a built-in means to perform channel assessment. Furthermore, in the evaluation of 13 commercial single chip transceivers [11]-[23] (conducted in this thesis), none possessed any self-contained, automated means with which to assess their relative operating spectrum. 
This thesis proposes hardware which can be integrated with existing single chip transceiver hardware to automate the channel assessment process with minimal impact on overall size and complexity. Chapter 2 outlines what is available in single chip transceiver technology for the MedRadio band and each receiver's hardware design. Chapter 3 discusses what an effective CCA system should comprise. Chapter 4 covers the design of evaluation hardware for a CCA system. Chapter 5 relates the performance of the CCA system. Chapter 6 examines how the CCA system could be extended or improved and Chapter 7 provides concluding remarks. 


\section{Single Chip Radio Technology}

Single chip transceivers were surveyed to understand the basic architectures employed in these devices. Each transceiver interfaces with a host microcontroller or microprocessor. The transceivers selected for evaluation operate within the frequency range of $401 \mathrm{MHz}$ to $457 \mathrm{MHz}$. This frequency range is covered by the MedRadio regulations [1], [2] (superseding the Medical Implant Communications Service [MICS] regulations) and provides fewer challenges to Radio Frequency (RF) circuit design when compared with higher frequencies such as the $915 \mathrm{MHz}$ and $2.4 \mathrm{GHz}$ industrial scientific medical bands. Manufacturers' datasheets were studied to determine specific elements that comprise each device's receiver as well as identify and evaluate key performance metrics. Datasheets often omit explicit details of implementation but provide sufficient information for this work.

\subsection{Receiver Structure}

Of the commercial transceivers studied all implement some form of directconversion receiver architecture [24]. A block diagram depicting the directconversion receiver is shown in Figure 1. The incoming RF signal is supplied to a pair of down-converting mixers for incident and quadrature, driven by a local oscillator (LO) signal at both $0^{\circ}$ and $90^{\circ}$ phase, respectively. The mixer output, the intermediate frequency (IF) signal, is then filtered and amplified by a variable gain amplifier (VGA) before being sampled by an analog to digital converter $(A D C)$. There is often feedback from the ADC to the VGA which allows the 
appropriate gain level to be set dynamically. In all of the transceivers studied, the LO signal is provided by either an integer- $\mathrm{N}$ frequency synthesizer or a fractional$\mathrm{N}$ frequency synthesizer. Table 1 gives a comparison of the receiver structures present in the transceivers evaluated.

\begin{tabular}{|l|l|l|l|l|l|l|l|l|}
\hline Manufacturer & Model \# & $\begin{array}{l}\text { Stage } \\
1\end{array}$ & $\begin{array}{l}\text { Stage } \\
2\end{array}$ & $\begin{array}{l}\text { Stage } \\
3\end{array}$ & $\begin{array}{l}\text { Stage } \\
4\end{array}$ & $\begin{array}{l}\text { Stage } \\
5\end{array}$ & $\begin{array}{l}\text { Stage } \\
6\end{array}$ & $\begin{array}{l}\text { Sample } \\
\text { Type }\end{array}$ \\
\hline TI & CC1120 & LNA & Mixer & $\begin{array}{l}\text { IF } \\
\text { Amp }\end{array}$ & Filter & -- & -- & ADC \\
\hline TI & CC1021 & LNA1 & LNA2 & Mixer & Filter & VGA & ADC & ADC \\
\hline TI & CC1020 & LNA1 & LNA2 & Mixer & Filter & VGA & ADC & ADC \\
\hline Micrel & MICRF506 & LNA & Mixer & $\begin{array}{l}\text { IF } \\
\text { Amp }\end{array}$ & $\begin{array}{l}\text { SK } \\
\text { Filter }\end{array}$ & Filter & -- & ADC \\
\hline $\begin{array}{l}\text { Analog } \\
\text { Devices }\end{array}$ & ADF7020-1 & LNA & Mixer & Filter & VGA & -- & -- & Limiter \\
\hline Nordic & nRF905 & LNA & Mixer & $\begin{array}{l}\text { IF } \\
\text { Filter }\end{array}$ & -- & -- & -- & ADC \\
\hline Atmel & ATA5428 & LNA & Mixer & $\begin{array}{l}\text { IF } \\
\text { Filter }\end{array}$ & $\begin{array}{l}\text { IF } \\
\text { Amp }\end{array}$ & -- & -- & ADC \\
\hline NXP & OL2381 & LNA & Mixer & $\begin{array}{l}\text { IF } \\
\text { Filter }\end{array}$ & $\begin{array}{l}\text { IF } \\
\text { Amp }\end{array}$ & -- & -- & Limiter \\
\hline Silicon Labs & Si4430/31/32 & LNA & Mixer & VGA & -- & -- & -- & ADC \\
\hline \multirow{2}{*}{ RFM } & TRC102 & LNA & Mixer & $\begin{array}{l}\text { IF } \\
\text { Filter }\end{array}$ & $\begin{array}{l}\text { IF } \\
\text { Amp }\end{array}$ & -- & -- & ADC \\
\hline RFM & TRC105 & LNA & Mixer & VGA & Filter & Filter & $\begin{array}{l}\text { IF } \\
\text { Amp }\end{array}$ & ADC \\
\hline Maxim & MAX7031 & LNA & Mixer & VGA & -- & -- & -- & Limiter \\
\hline Maxim & MAX7030 & LNA & Mixer & VGA & -- & -- & -- & Limiter \\
\hline
\end{tabular}

Table 1 - A comparison of the apparent architecture of 13 commercial chip transceivers.

Note: LNA = low noise amplifier

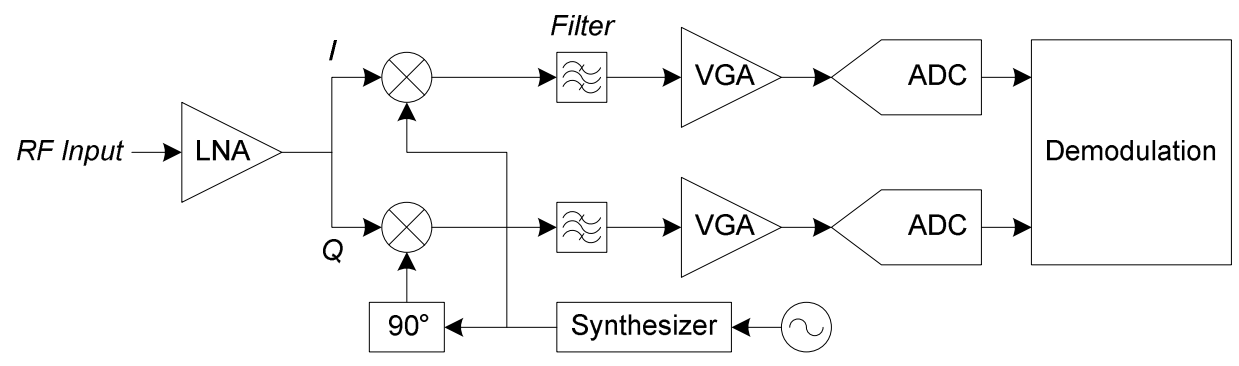

Figure 1 -The direct-conversion reciever architecture. 
Several transceivers do not sample the down-converted signal directly but instead amplify the signal through a chain of low gain VGAs referred to as a limiter chain [25]. At each stage, the signal level is assessed and feedback is provided to set the gain level. Once the signal has achieved the required voltage level, it is supplied directly to the demodulation hardware. Figure 2 shows an example of such a limiter chain. In this receiver architecture, the received signal strength indicator (RSSI) is proportional to the level of gain supplied by the VGA or limiter chain. The RSSI level is used to assess the relative power of the received signal.

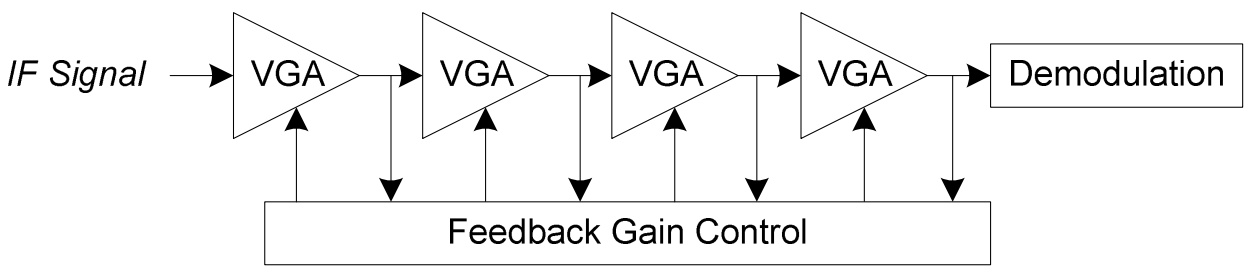

Figure 2 - Limiter amplifier chain for receiver gain control.

\subsection{Power Usage in Single Chip Transceivers}

These transceivers are targeted primarily at low power, low cost, personal electronics applications. Many of these applications require battery operation and place significant emphasis on low power operation. The transmit section of these devices will contribute significantly to the overall power consumption but is outside the scope of this research. All of these devices have three or four operation modes consisting of receive ( $R X)$ mode, transmit (TX) mode, 
shutdown, or idle mode where the frequency synthesizer runs continuously to minimize startup delay. Table 2 was constructed with data from the manufacturers' datasheets to illustrate which section of the receiver contributes to the overall $\mathrm{RX}$ mode power consumption.

\begin{tabular}{|l|l|r|r|r|r|r|r|}
\hline \multicolumn{1}{|c|}{ MFG } & \multicolumn{1}{|c|}{ Model \# } & $\begin{array}{c}\text { Lowest } \\
\text { Current } \\
(\mathrm{mA})\end{array}$ & \multicolumn{1}{c|}{ Delta } & $\begin{array}{c}\text { Highest } \\
\text { Current } \\
(\mathrm{mA})\end{array}$ & $\begin{array}{c}\text { w/Synth } \\
\text { Running } \\
(\mathrm{mA})\end{array}$ & $\begin{array}{c}\text { w/Crystal } \\
\text { Current } \\
(\mathrm{mA})\end{array}$ & $\begin{array}{c}\text { No } \\
\text { Crystal } \\
\text { Current } \\
(\mathrm{mA})\end{array}$ \\
\hline $\mathrm{TI}$ & CC1120 & 17.000 & 5.000 & 22.000 & -- & 1.300 & 0.170 \\
\hline $\mathrm{TI}$ & CC1021 & 19.900 & 0.000 & 19.900 & 7.500 & 0.500 & 0.077 \\
\hline TI & CC1020 & 19.900 & 0.000 & 19.900 & 7.500 & 0.500 & 0.077 \\
\hline Micrel & MICRF506 & 8.000 & 4.000 & 12.000 & -- & 0.780 & 0.280 \\
\hline Analog & ADF7020-1 & 17.600 & 2.500 & 20.100 & -- & 2.000 & -- \\
\hline Nevices & nRF905 & 10.500 & 2.000 & 12.500 & -- & 0.100 & 0.013 \\
\hline Atmel & ATA5428 & 10.500 & 3.500 & 14.000 & 8.800 & 0.430 & 0.370 \\
\hline NXP & OL2381 & 15.500 & 2.500 & 18.000 & 5.000 & 0.900 & 0.005 \\
\hline Silicon Labs & Si4430/31/32 & 18.500 & 0.000 & 18.500 & 8.500 & 0.800 & 0.450 \\
\hline RFM & TRC102 & 11.000 & 0.000 & 11.000 & -- & 0.600 & -- \\
\hline RFM & TRC105 & 2.700 & 0.000 & 2.700 & 1.400 & 0.065 & 0.001 \\
\hline Maxim & MAX7031 & 6.700 & 2.000 & 8.700 & -- & -- & 0.008 \\
\hline Maxim & MAX7030 & 6.400 & 1.900 & 8.300 & -- & -- & 0.008 \\
\hline Average & & 12.631 & 1.800 & 14.431 & 6.450 & 0.725 & 0.133 \\
\hline Standard Deviation & 5.533 & 1.646 & 5.603 & 2.567 & 0.524 & 0.155 \\
\hline
\end{tabular}

Table 2 - The stated current consumption for various operating modes for each of the chip transceivers studied.

Across the population, the average current consumption of the shutdown and idle modes were $0.133 \mathrm{~mA}$ and $6.450 \mathrm{~mA}$, respectively. In receive mode, some variability is noted in current consumption primarily from the amount of gain required in the receive chain. The average peak $\mathrm{RX}$ current was $14.431 \mathrm{~mA}$ and the average minimum $\mathrm{RX}$ current was $12.631 \mathrm{~mA}$ with an average difference of $2.340 \mathrm{~mA}$. Of the available idle mode current data, an average value of $6.450 \mathrm{~mA}$ 
was calculated for the population. This indicates that slightly less than half of the $\mathrm{RX}$ mode current is from frequency synthesis and that the variability of supplied current in the VGA chain is only about $16 \%$ of the peak $R X$ mode current.

\subsection{Channel Assessment}

To help improve the probability of error-free communication and reduce power consumption, communication protocols may rely on CCA to find channels with the lowest levels of noise [4]. This is referred to as listen-before-talk (LBT) in the MedRadio regulations. Some protocols may employ carrier sense multiple access [26] in which a mote or node in a wireless network must contend for available channels with other cooperative or competing nodes. As with any communication channel, it is also possible for time-varying levels of noise to exist as well. A channel assessment mechanism could also be used to facilitate a channel scan when a node is searching for a cooperative node with which to communicate.

The most straightforward means to perform CCA is to simply enter RX mode and listen for the expected signals. If no intelligible data can be found, an RSSI level can be used to determine if a noise source or competing signal is present in the channel. A high RSSI level would suggest the presence of such a signal. There are several downsides to this method. First, entering receive mode to simply listen for a clear channel requires just as much current as receiving useful packet data. For receivers with dynamic gain control, more current is required to listen to channels with low to no signal levels as compared with channels where there is a 
strong, potentially identifiable signal. Another issue is that, for the majority of the transceivers studied, no automated assessment mechanism exists. This requires the host controller to be fully active, listening for received data and managing channel switching as well as RSSI level assessment. This further increases current consumption and busies the controller with additional overhead.

The only transceiver reviewed in this study that had any form of automated channel assessment capability was the Silicon Labs Si443x. To provide this function, the Si443x has a single register which complements the RSSI register into which a threshold value can be written. The controller can set this threshold level and place the transceiver into receive mode. The controller is then free to enter a low power state. If the RSSI level exceeds the threshold level, an interrupt mechanism can be triggered to wake the controller. This is a small improvement but only benefits the overall current consumption by allowing the controller to enter a lower power state and restricts the assessment to a single channel. 
3. CCA System Requirements

In order to design an effective CCA system, it is critical to understand the types of signals that may be present in the frequency band of interest. These include not only wanted signals but unwanted signals as well. Unwanted signals can take the form of noise or excessive power from devices operating in adjacent bands or at harmonic multiples of the operating band of interest. Devices operating the MedRadio frequency bands have well-defined operating characteristics.

\subsection{MedRadio Requirements}

MedRadio devices typically use medium to low data rate digital communication with a frequency shift key (FSK) or amplitude shift key (ASK) modulation scheme. Phase shift key (PSK) modulation is possible but, based on the commercial transceiver offerings, not typically available. The MedRadio regulations set limits on the power levels and the occupied bandwidth of transmitters operating in the various bands within the $401-457 \mathrm{MHz}$ range. MedRadio devices operating in the 401-406 $\mathrm{MHz}$ range have a bandwidth limitation of up to 300 kilohertz $(\mathrm{kHz})$ from $402-405 \mathrm{MHz}$ and $100 \mathrm{kHz}$ otherwise. The MedRadio requirements also dictate that devices operating in the 402-405 $\mathrm{MHz}$ band define the center channel about $403.65 \mathrm{MHz}$.

The LBT requirements for MedRadio devices state that transmitters operating in the $402-405 \mathrm{MHz}$ band must listen for $10 \mathrm{~ms}$ per channel, five seconds prior to any attempt to transmit on that channel. The listening device must register a 
channel in use if the power detected in the channel exceeds a threshold given by Equation 1.

$$
10 \log _{10} B(H z)-150\left(\frac{d B m}{H z}\right)+G(d B i)
$$

Equation 1 - Detection threshold of occupied channels in the $402-405 \mathrm{MHz}$ band.

Here $B$ represents the widest emission bandwidth of the signal to be transmitted and $G$ represents the isotropic antenna gain of the transmitter. For an FSK signal with a single-sided frequency deviation of $50 \mathrm{kHz}$ and a Manchester encoded bit rate of 48 kilobits per second (kbps), the estimated bandwidth is approximated by Carson's Rule (Equation 2) [27]. If the antenna gain is assumed flat over the $402-405 \mathrm{MHz}$ band at $0 \mathrm{dBi}$ ( $\mathrm{dB}$ relative to an isotropic radiating source) the LBT detection threshold is shown in Equation 2.

$$
\begin{aligned}
& B=2 *\left(\Delta f+f_{m}\right) \\
& 10 \log _{10}(2 *(50000+48000))-150+0=-97 d B m
\end{aligned}
$$

Equation 2 - Carson's bandwidth rule and the resulting detection threshold.

\subsection{Channel Assessment Time}

There are no access time restrictions in the MedRadio bands. Once a channel is established between two devices, the communication session is permitted to proceed indefinitely. However, endless communications are unlikely. It is reasonable to assume that the majority of communications will consist of short bidirectional sessions between two or more nodes. Consider a three-part 
bidirectional session between two nodes consisting of an initiation packet (192 encoded bit periods), a data packet (576 encoded bit periods), and an acknowledgement packet (192 encoded bit periods). The Manchester encoded bit rate will be $48 \mathrm{kbps}$.

Figure 3 shows the communication structure and timing for a simple two node network. It is assumed that there is no delay for each endpoint's transition between receive mode and transmit although the actual delay may be some number of bit periods. The Analog Devices ADF7020-1 transceiver datasheet specifies this turn-around time to be roughly $252 \mu$ s at 48 kbps but this time varies by manufacturer.

\begin{tabular}{|c|c|c|c|}
\hline \multirow{2}{*}{$\begin{array}{l}\text { Master } \\
\text { Sensor }\end{array}$} & INIT (192) & Receiving & ACK (192) \\
\hline & Receiving & DATA (576) & Receiving \\
\hline me (ms) & & & \\
\hline
\end{tabular}

Figure 3 - Hypothetical, three-part, bidirectional protocol for a two-node MedRadio network

Assuming a hypothetical arrangement of 25 of these two-node networks, and each individual node has effectively equal probability of detecting any other transmitting node in the listening space. Each pair of nodes is comprised of a sensor node and a master node that attempts to obtain data from the sensor node every five minutes on a randomly selected channel. If the INIT packet fails to garner a response, the master node does not retry but simply waits for another five minutes to elapse, disregarding the time spent with its failed attempt. 
Assuming the INIT packet has 80 encoded bit periods of preamble, this protocol provides a $1.667 \mathrm{~ms}$ window in which a sensor node is able to detect and possibly decode an INIT packet from its paired master node. In contrast, an unpaired sensor or master node has a $20 \mathrm{~ms}$ window in which this exchange between any other two-node networks could be detected. This illustrates that even with nodes that maintain synchronization between communication attempts, a CCA mechanism that is able to quickly scan all available channels could aid in improving network performance by not only avoiding blocked channels but quickly identifying potentially paired nodes as well. An effective channel assessment mechanism must to be fast enough to allow for scanning that can capture these short duration events.

\subsection{Channel Assessment Protocol}

There are two basic functions of the channel assessment mechanism. The first is to identify the least-interfered channel in accordance with an LBT protocol for a node that wishes to transmit. The intuitive choice would be to measure the power level directly at the center of each channel. This is true for ASK modulation but the spectral distribution for an FSK signal would have the highest power level concentration at some frequency deviation above and below from the carrier frequency. The second function of the channel assessment system is to allow a receiving node to make a best guess as to which channel to begin listening by determining if there is a signal centered within the channel of interest. The challenge then is that, with only measuring in the center of the channel, it 
would be possible to see a lower power level from a slightly off-channel signal as shown in Figure 4(a).

In both cases, the assessment should be flexible enough to provide a sufficient number of measurement points to make a conclusive judgment. For the LBT transmitter case, it may be sufficient to measure at two points about the center of each channel. For the receiver case, three points per channel may be effective. For some receiver cases, it may be possible to measure the power level at the boundary of two channels thus reducing the number of measurement points per channel as shown in Figure 4(b). Both scenarios described in Figure 4 assume a nearly ideal selectivity of the down conversion stage. The effect of non-ideal down-conversion will be discussed in the next section.

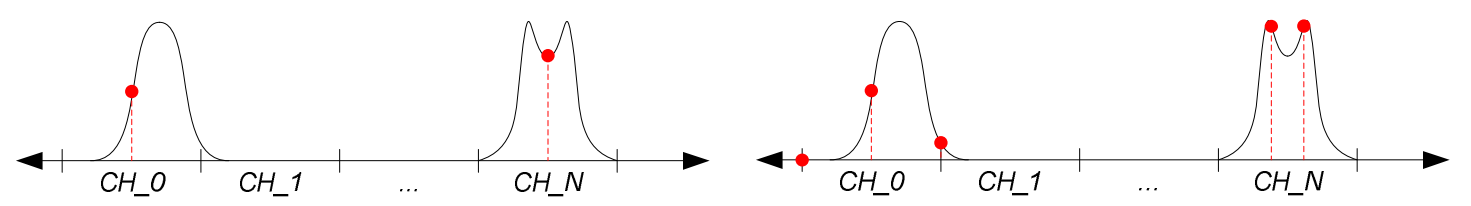

(a)

(b)

Figure 4 - Potential measurement points within a channel. 


\section{Channel Assessment Means}

Because MedRadio devices primarily use FSK or ASK modulation and have a limited channel bandwidth between 100 and $300 \mathrm{kHz}$, the spectral distributions should be fairly well-defined. Detection of such a signal should be relatively simple as well. A simple square-law power detector circuit could be employed. In fact, this method is a typical means with which an RSSI level can be extracted in a receiver [27]. In order to assess the in-channel power, it is important to isolate the signal in the channel and avoid the influence of adjacent channels on the measurement. One option is to use very selective filtering, restricting the outof-channel signals. The problem with very narrow, multi-pole, band-pass filters is that the signal of interest can be greatly attenuated, calling for additional amplification after filtering. From a low power detection standpoint, this is fairly wasteful.

Another option is to down-convert the channel of interest, filter at the IF stage, and finally measure the power of the IF signal. The direct-conversion style receiver architecture shown in Figure 1 contains most of the elements required to do this already and is employed by many of the single-chip transceivers discussed above. A similar system has been proposed in [28] but is designed as a standalone system and does not take advantage of the existing resources of a chip transceiver. 


\subsection{Down-Converting Mixer}

A mixer can be constructed in many ways but can be categorized into two basic types, active and passive. Active mixers are typically constructed using field effect transistors [29], but some forms may also employ bipolar junction transistors or commercially available complementary metal oxide semiconductor processes [8]. Active mixers limit the losses incurred in down-conversion process and, in some cases, boost the IF signal output level. The downside of active mixers is that they require biasing or amplification which, in turn, requires some amount of power. Passive mixers can be constructed with simple diode-based structures and require no biasing in most cases. This allows passive mixers to be much more power efficient but at a cost of higher local oscillator (LO) drive levels to reduce the conversion loss (reduction in IF output power) [29]. For this study, a doubly balance mixer [29], comprised of a schottky diode bridge and two balun transformers, was selected for simple construction and straightforward performance characterization.

One limitation to this mixer design is that it is a double-sideband (DSB) mixer [27] with no means to suppress frequency images that may intersect the IF filter bandwidth. Figure 5 shows that, as the LO signal to the mixer is stepped through the band of interest, any signal above the LO frequency within the IF filter bandwidth will be present in the down-converted signal spectrum. As the LO frequency steps past this signal, the image is present in the IF filter bandwidth. The transceivers considered in this study use a single-sideband (SSB) [27] or 
image-rejection mixer [30] as shown in Figure 1. This eliminates the image problem.
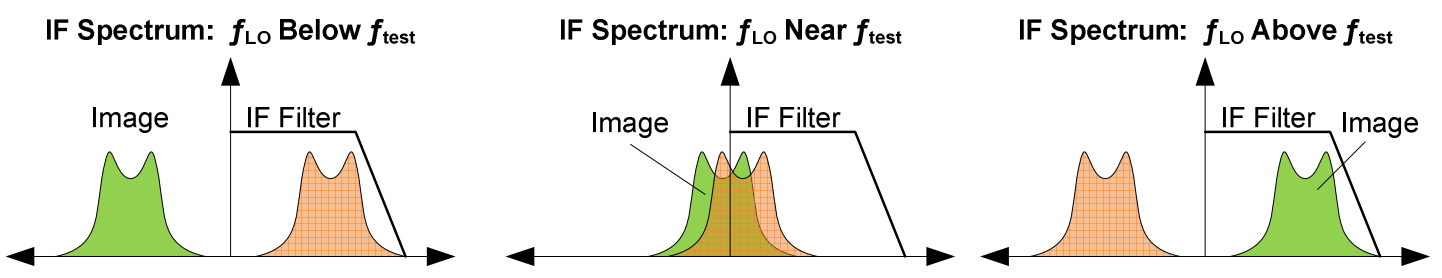

Figure 5 - An unsuppressed image signal from a DSB mixer appearing in the IF filter bandwidth.

\subsection{Frequency Source}

The commercial transceivers studied use some form of frequency synthesizer to produce the LO signal for the down-conversion stage in the receiver chain. Typical frequency synthesizers are phase-lock-loop (PLL) based. PLLs use a low frequency crystal oscillator reference in a phase detection loop to control a voltage controlled oscillator (VCO) which generates the higher frequency LO signal [31]. The control of the frequency is through a set of $\mathrm{N}$-integer or fractional-N dividers. PLL synthesizers have poorer noise performance than high frequency crystal oscillators but are usually much cheaper to implement. For this application, the noise considerations are not as stringent because the assessment of power levels between channels is relative. For simplicity, a simple monolithic VCO was selected as the frequency source. To attain sufficient drive level, the VCO output will be amplified before it is supplied to the mixer stage. 


\subsection{Power Detector}

The mixer stage translates the RF signal content around the LO frequency to an IF signal. The IF signal will therefore constitute a representation of all signal power near the LO frequency. Many transceivers employ an IF power detector to assess RSSI through an RF coupled path [31]. Because the primary concern is measuring the IF signal power without demodulation, the IF output of the mixer is directly coupled to a small-signal IF detector circuit [31] which supplies a DC signal whose output can then be sampled. In order to ensure the signal that reaches the detector circuit is only the IF signal of concern, a single-pole lowpass filter allows frequencies below $300 \mathrm{kHz}$ to pass to the detector.

\subsection{Control Output and Measurement}

In an actual receiver, there are often many functions under some form of digital control including frequency selection, amplifier gain, filter selectiveness, and many demodulation parameters. In this application only control over the LO frequency is required. A 12-bit digital to analog converter (DAC) was used to digitally control the tuning voltage for the VCO. The receiver also requires a means to translate the DC voltage output of the detector circuit to a digital representation so it can be provided to the receiver controller. This is achieved through a 12-bit ADC. 


\subsection{Digital Control}

In a single chip transceiver, the various functions of the device are managed by a digital control block. The purpose of this block is to control the operating parameters of various functional blocks within the transceiver, handle digital modulation/demodulation, and provide control feedback to the host controller. The structures in the digital block could include register files, state machines, and control of ADCs and DACs used to monitor and control the transceiver functional blocks. For this application, the digital block is simply required to manage the ADC input control, the DAC output control, registers, and the channel assessment state machine. To approximate the digital block of a chip transceiver, a field programmable gate array (FPGA) was used. A block diagram of this system is shown in Figure 6.

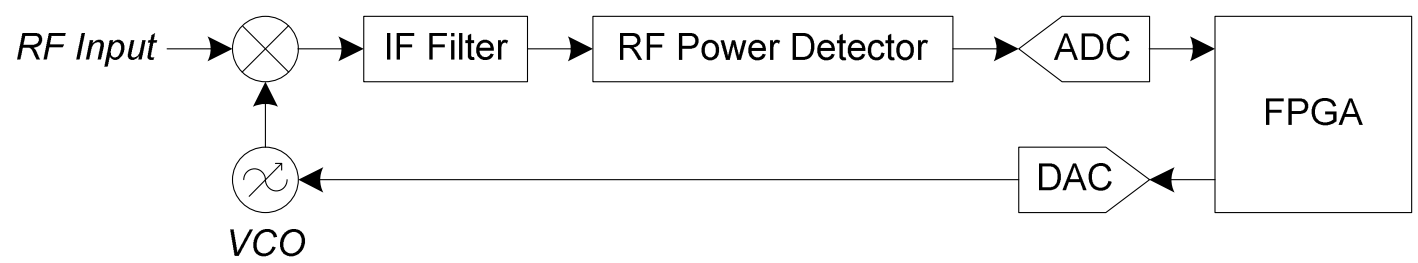

Figure 6 - Block diagram of the study system

The ADC and DAC are both controlled over a serial bus protocol, unlike a true digital control block where the ADC and DAC typically have parallel read/write registers. One functional block in the FPGA controls these serial interfaces in a transparent way that will make them appear as parallel interfaces to the remaining digital blocks. The channel assessment mechanism consists of a set 
of registers which will serve as a list of frequency points where the power level is measured, a state machine that controls the measurement and frequency selection process, and result registers. The frequency control registers will contain the DAC values that will control the VCO tuning voltage.

To trigger a scan sequence, the Start Scan register is written to $0 \times 01$. This register is then cleared by hardware. The state machine then sets the DAC value, allows time for the $\mathrm{VCO}$ to settle, initiates the ADC conversion, reads back the result from the ADC, and places the result in the result registers for each channel sequentially. To observe the data recovered by the channel assessment system, a universal asynchronous receiver/transmitter (UART) interface from the FPGA to a PC will be used. A diagram of the digital block can be found in Figure 7 and the state flow diagram in Figure 8. The channel assessment mechanism could be implemented in two ways, shown in Figure 7. One method is to simply provide an ADC value for each channel which can be then be processed by the host controller. This has the disadvantage of requiring an additional register in the register file for each channels sample. If multiple scans are used between host controller reads, a significant number of registers would be needed. A more simplistic but less resource constraining method is to have the CCA system automatically test each sample against a "Threshold" register. As each ADC sample is returned, it is tested against the threshold value. If the ADC sample is greater than the threshold value, a single bit is set for the corresponding channel in the CCA result register(s). Only a single bit would be required for each 
channel but multiple registers may need to be used depending on the number of channels.

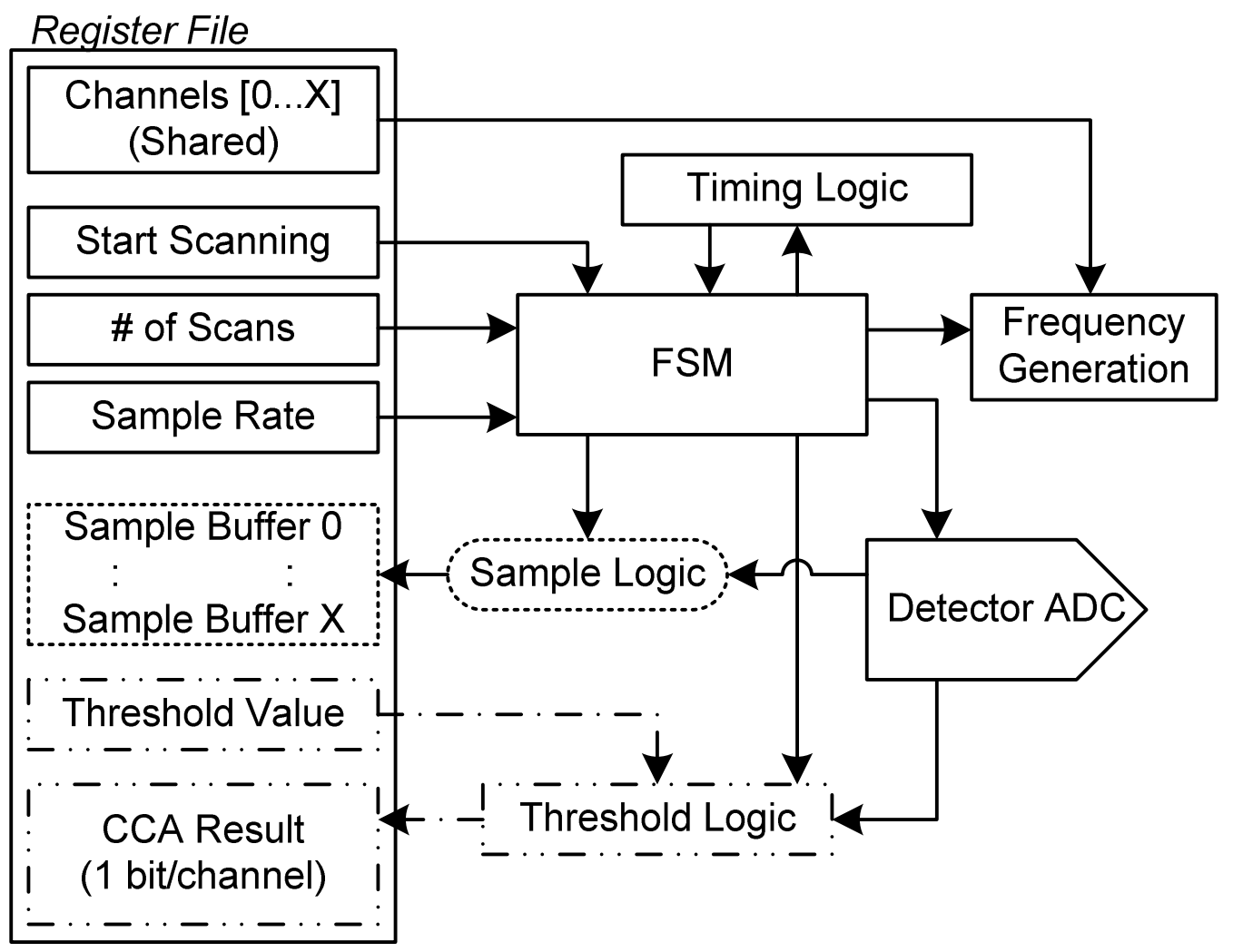

Figure 7 - Block diagram of the digital control hardware. 


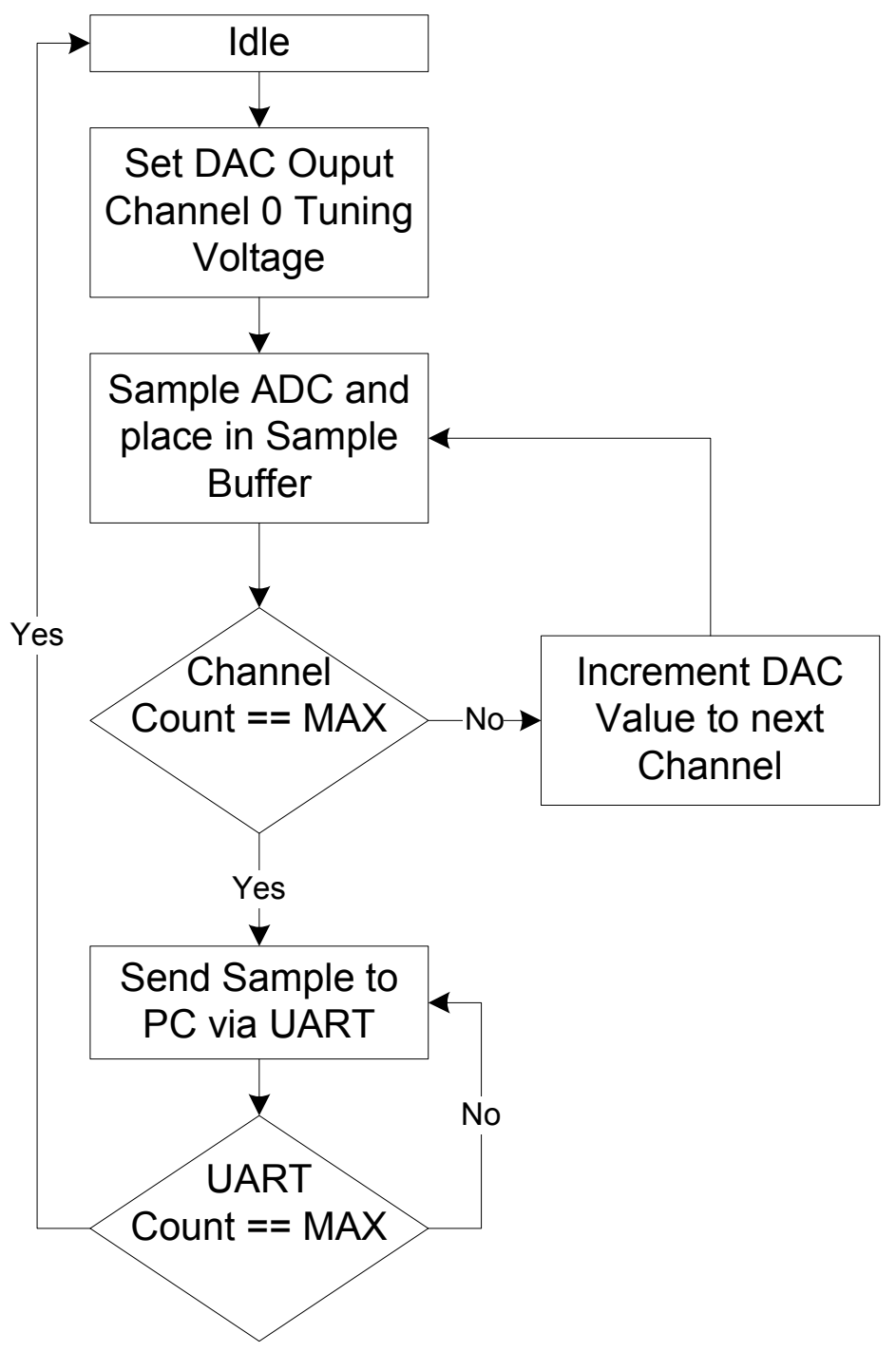

Figure 8 - State flow diagram of the hardware finite state machine (FSM).

4.6. Hardware Impact on Transceiver Size

The impact on the transceiver die area requirement is worth considering. A transceiver is a complex system consisting of both analog and digital elements as well as consideration for resonant structures used in the frequency synthesis and filtering. For example, a MICS transceiver has been implemented on a $1.9 \mathrm{x}$ $2.5 \mathrm{~mm} 2$ die using a $0.18 \mathrm{um}$ CMOS process [7]. For this design an estimated 
$9 \%$ of the overall die size is devoted to the digital controller. Because the hardware changes required for a CCA system should be mainly in the digital controller it is reasonable to assume the impact on overall die size should be small. The digital portion of the transceiver will already contain registers, timing, and control logic. If we assume that the CCA system will increase the digital block size by $10 \%$ this will translate to an overall die size increase of less than $1 \%$.

\subsection{CCA System Hardware}

The FPGA used for the evaluation was a Xilinx Spartan 6 on a Digilent Nexys 3 development board [32]. A custom printed circuit board was designed to interface the other hardware with the FPGA. The DAC used was a Texas Instruments DAC121S101 [33] which is a 12-bit, single-channel DAC with built-in output buffering. The ADC used was an Analog Devices AD7991 12-bit, 4channel ADC [34]. Each ADC input was buffered using an Op-Amp in a $20 \mathrm{kHz}$ low pass filter configuration. The VCO was a Maxim MAX2608 [35] matched to an Avago MGA-68563 [36] low noise amplifier. This combination provided sufficient signal drive $(+7 \mathrm{dBm})$ to the LO input of the mixer in the $402-405 \mathrm{MHz}$ band. The doubly balanced mixer was comprised of two, Mini Circuits TCM4-19+ wire wound balun transformers [37] and a Skyworks SMS3626-023 Schottky diode quad [38]. The IF low pass filter used was a single pole LC. The detector circuit used a single Skyworks zero biased Schottky detector diode. A picture of the CCA evaluation system is shown in Figure 9. 


\subsection{Hardware Timing Considerations}

The DAC (TI DAC121S101) and ADC (Analog Devices AD7991) used in the CCA system both have serial interfaces with limited bus speeds. These bus speeds impose significant timing constraints which would not exist in a system employing parallel read/write hardware that is likely to be found in most transceiver architectures. The critical timing limit is imposed by the ADC which limits the sample rate to 2,500 samples per second. For this reason, the CCA system is able to sample a new channel every $0.8 \mathrm{~ms}$. As discussed in section 3.3, the number of points used to assess each channel dictates the ability of the CCA system to localize detected energy within the frequency band. Because the channel scan rate is fixed, increasing the number of channels in the scan will increase the total scan time. This effectively increases the probability for very short packets to be missed by the scan protocol. The proposed block diagram shown in Figure 7 is not completely represented by the evaluation system. The CCA system does not contain "Start Scanning" or "\# of Scans" registers and instead a $0.5 \mathrm{~s}$ overflow timer triggers each scan in an endless loop. 


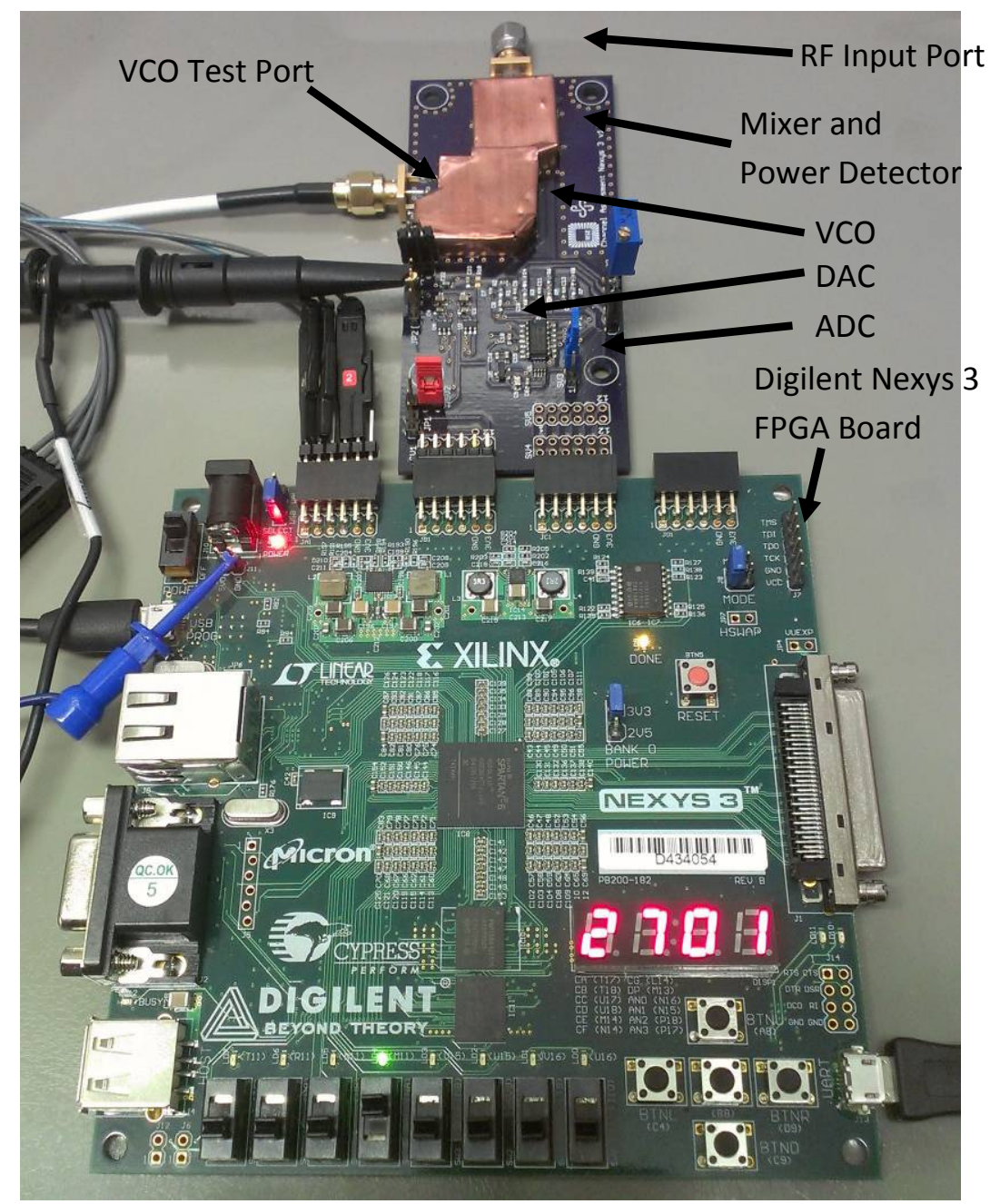

Figure 9 - CCA System hardware

4.9. Signal Power Level and Power Consumption Considerations In order to accurately assess the power in a given channel, a CCA system must have a well characterized output response within the band of interest. The actual output of a power level detector will depend on several factors including low noise amplifier gain, mixer conversion gain (or loss) as well as the losses associated with filtering and noise suppression. This level of hardware performance is outside the scope of this work as the major thrust is to show the 
potential of such a CCA system. The same can also be said for system power consumption. Precise characterization of the input power to output result value will not be undertaken. It is assumed that the frequency response of the CCA system over the band of interest is linear and that the CCA system will have sufficiently low noise levels to provide meaningful results. The actual implementation of such a system is left to the transceiver designer. 


\section{CCA System Performance}

To evaluate the performance of the CCA system a test strategy was devised. Several tests were conducted demonstrating the proper operation of each portion of the design and CCA system behavior to the presence of common RF signals.

\subsection{CCA Test Setup and Conditions}

To test the operating capabilities of the CCA system proposed in section 4 , the test setup shown in Figure 10 was used. The SMIQ 03B signal generator was used to create various modulated signals within the test band of interest (402$405 \mathrm{MHz}$ ). A spectrum analyzer was used to capture the output of the signal generator for later comparison with the results reported by the CCA system. As each scan completes, the stored ADC samples are truncated to 8-bits and written to the UART. Each scan sample set is collected on the PC side by a Python script and stored in a comma separated value file for evaluation. Additionally, software (Figure 11) was written to control the SMIQ for packet tests. The CCA system evaluated does not employ the automatic assessment means discussed in section 4 as this would be trivially done in hardware and obtaining the raw sample data provides a better illustration of the system behavior.

The evaluation of the CCA system was broken into two test categories. One category characterizes the hardware response of the system to various modulated waveforms. The other evaluates the systems response to packet detection under various conditions. The CCA system was designed to sample 12 
or 34 channels, either of which could be selected while in operation through a hardware switch.

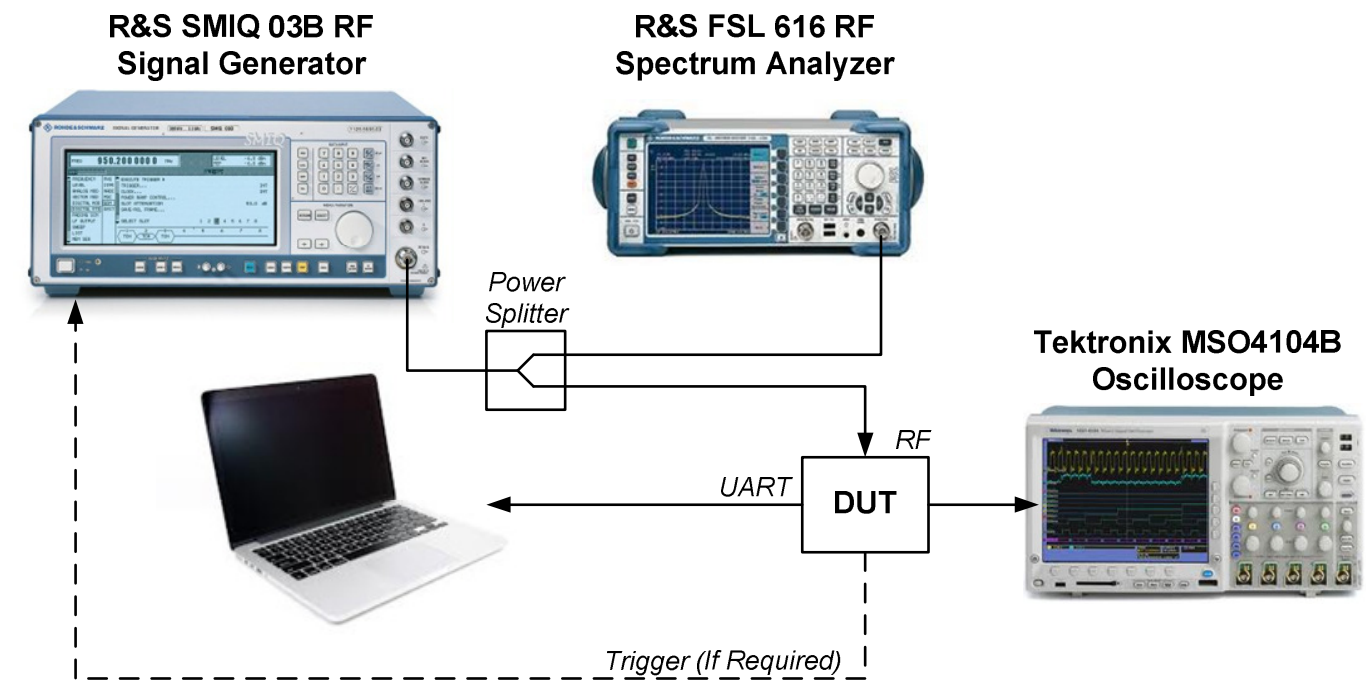

Figure 10 - Test setup used to evaluate the proposed CCA system. 


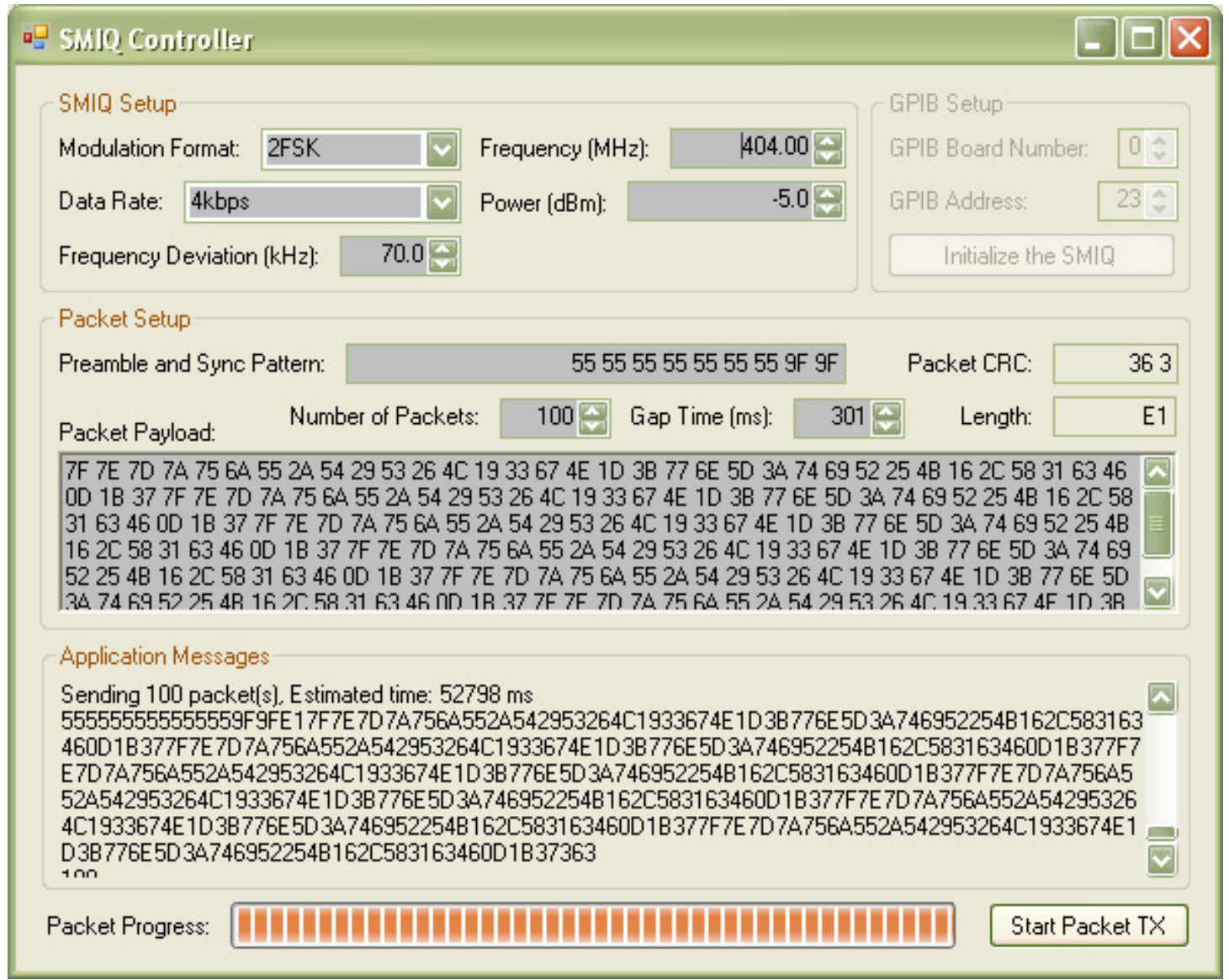

Figure 11 - SMIQ controller software written in C\# for packet testing.

5.2. Timing Confirmation

To confirm timing of the ADC and DAC as well as the VCO control voltage, screen captures of these signals were taken using both 12 and 34 channels as shown in Figure 14 and Figure 15. Figure 12 and Figure 13 show the accumulated frequency spectrum for the VCO output in both 12-channel and 34channel modes respectively. The screen capture for the 12-channel operation is a $4 \mathrm{~ms}$ per division resolution and shows discrete steps in the VCO tuning voltage (lower trace) indicating larger frequency channel steps. The 34 channel 
screen capture is at $10 \mathrm{~ms}$ per division and shows a much finer resolution control of the VCO tuning voltage. Both screen captures are at the same vertical scale. The flat portion at the top of each VCO tuning curve is the period in which samples are written out via the UART before the DAC value is reset to the starting channel. The upper two traces show write trigger timing for each DAC write and write completion.

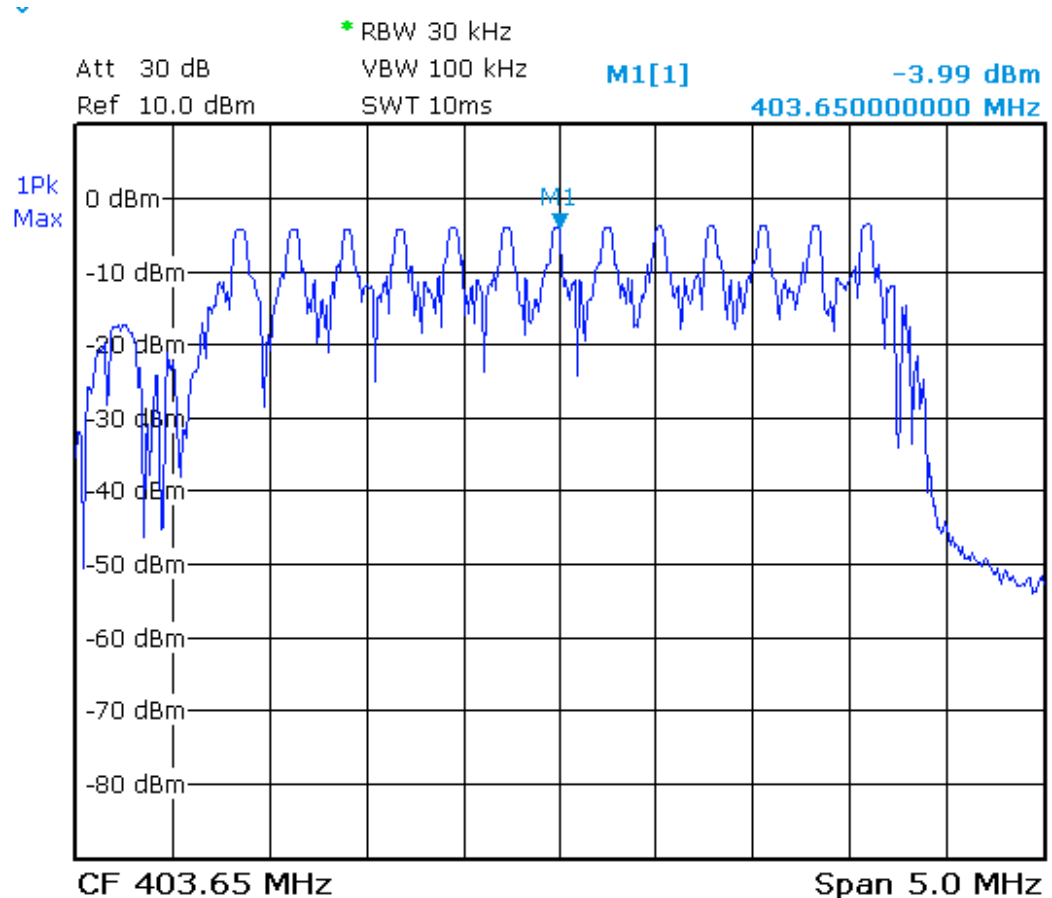

Figure 12 - Accumulated spectrum for the VCO output with 12 channels. 


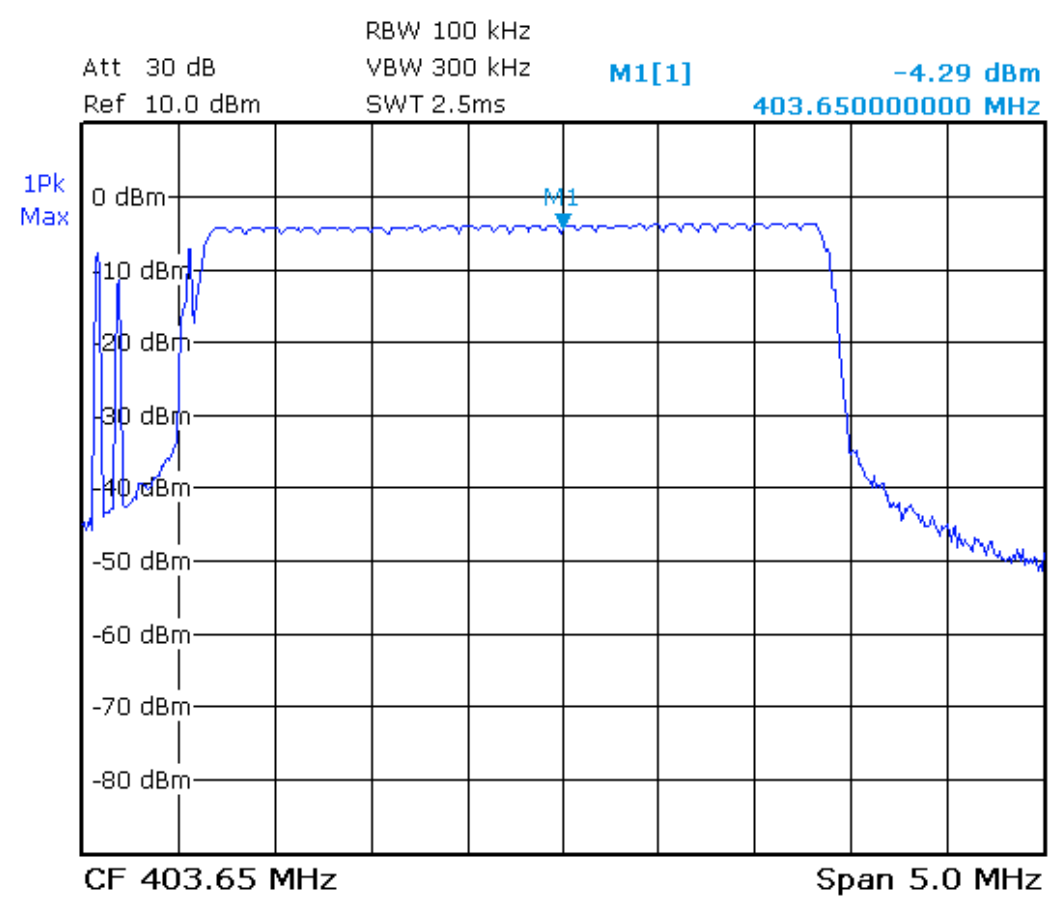

Figure 13 - Accumulated spectrum for the VCO output with 34 channels. 


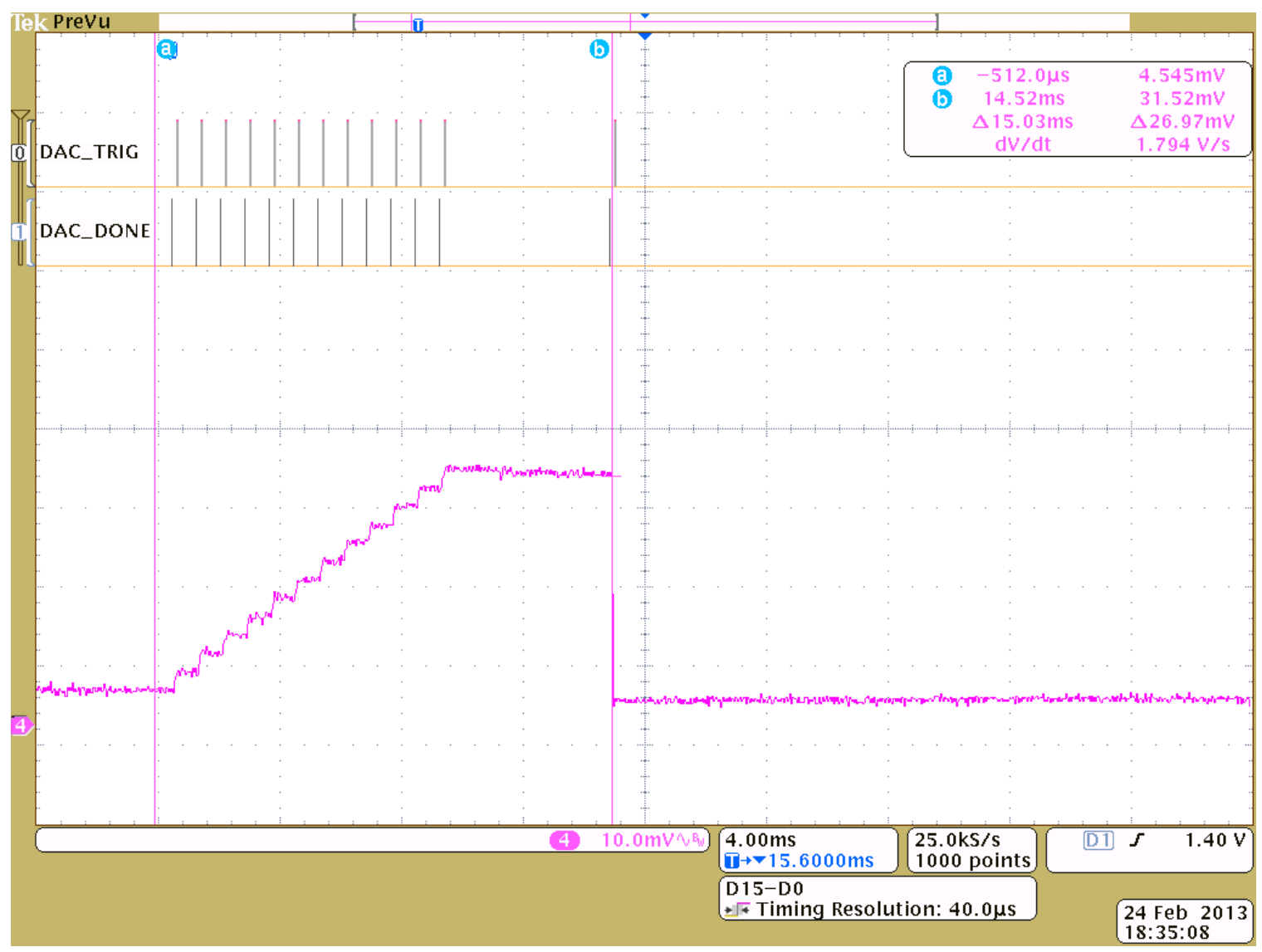

Figure 14 - DAC/ADC write/read timing and VCO output using 12 channels. 


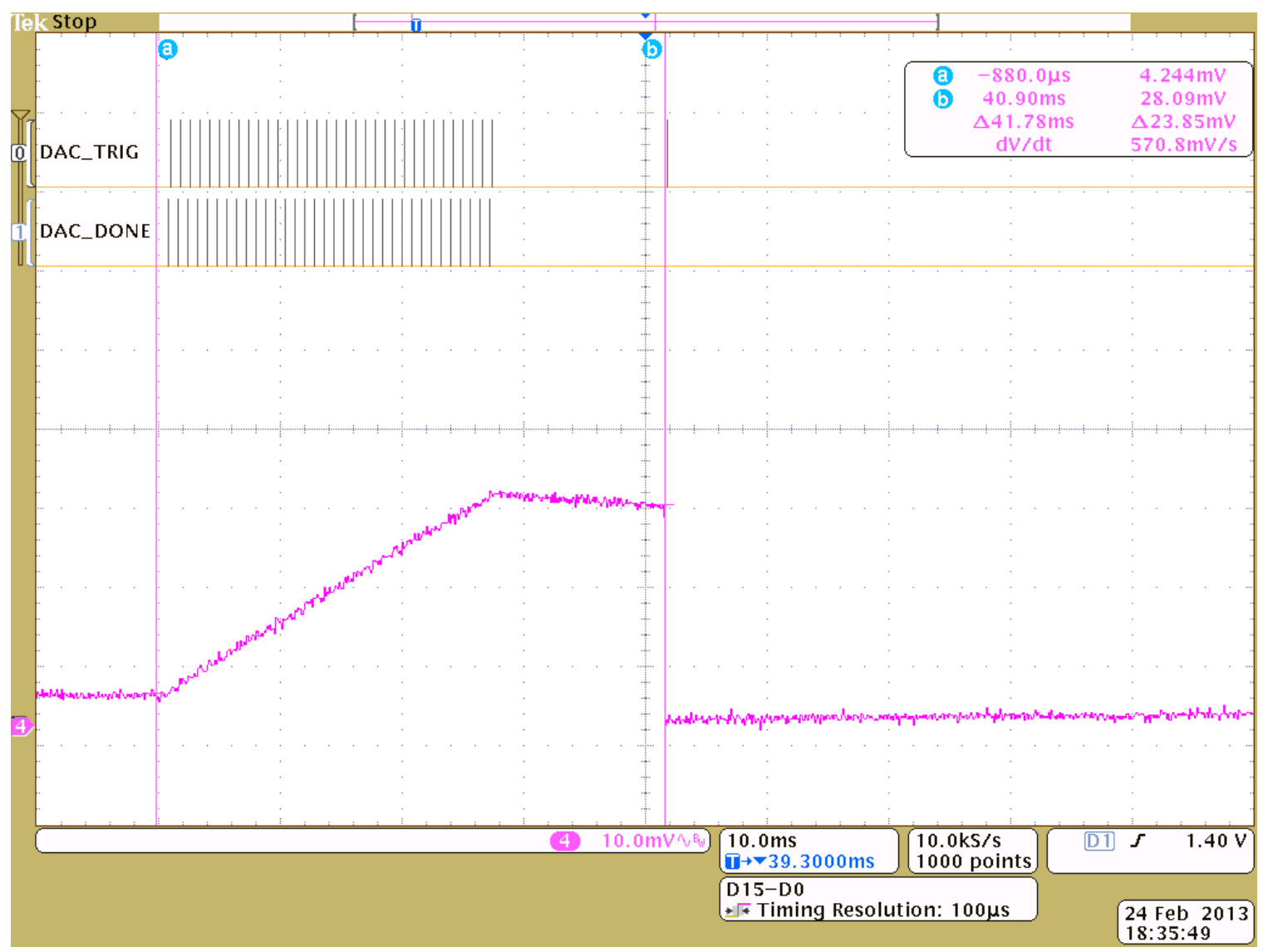

Figure 15 - DAC/ADC write/read timing and VCO output using 34 channels.

\subsection{Characterization Results}

Three signals were used to characterize the response of the CCA system to various signals that may be present in the frequency band of interest. The first was a wide band code division multiple access (WCDMA) modulated signal at a symbol rate of $1.8 \mathrm{M}$ symbols per second at a center frequency of $403.65 \mathrm{MHz}$. This would allow the CCA system to be characterized in the presence of a wide band signal. The second test signal was a Bluetooth modulation standard signal 
at a symbol rate of $1 \mathrm{M}$ symbols per second, a frequency deviation of $160 \mathrm{kHz}$, and centered about $403.65 \mathrm{MHz}$. The final test signal was a 2FSK signal with a frequency deviation of $10 \mathrm{kHz}$ and a symbol rate of $4 \mathrm{k}$ symbols per second centered about $403.65 \mathrm{MHz}$. This signal provides characterization of the CCA system for a very narrow bandwidth signal.

Figure 16 shows the spectrum analyzer capture for the WCDMA signal alongside the CCA results for both 12 and 34 channels. The spectrum analyzer capture shows the evenly dispersed energy over a $2 \mathrm{MHz}$ bandwidth with a roll off in power in both sides of about $0.25 \mathrm{MHz}$. The CCA system captures roughly the same spectral distribution which much greater detail provided in the 34 channel assessment. Because the energy in the WCDMA signal is spread over a larger bandwidth the power observed in the IF stage by the CCA system is relatively low for each bin. For this design, the IF signal level is just above the minimum for detection.

The Bluetooth modulation standard test signal and the associated CCA system responses are shown in Figure 17. The spectral distribution is narrower than the WCDMA signal in part due to the Gaussian FSK format but still requires a significant amount of bandwidth. With this test signal, the effect of the SSB mixer is visible. This is more apparent in the 34-channel CCA sweep than the 12channel sweep which makes the energy more difficult to localize. If a DSB mixer was used, this would not be an issue. The narrow 2FSK test signal in Figure 18 displays the SSB mixer issue clearly, creating what appear to be two energy 
sources in the band. If this image is ignored, the energy appears to be properly identified at about $403.7 \mathrm{MHz}$. All three test signals demonstrate that the CCA system accurately identifies channels in which no energy exists.

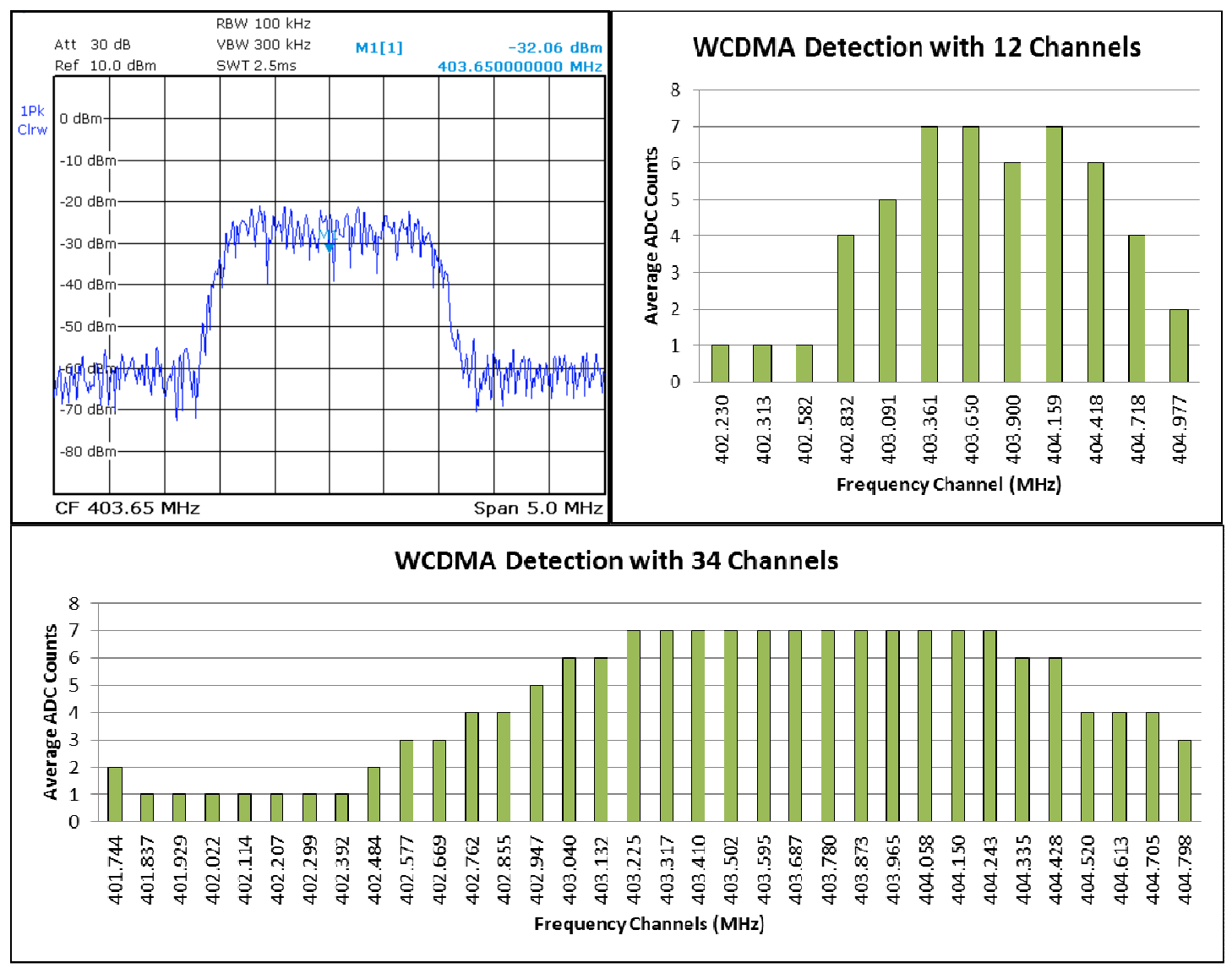

Figure 16 - The WCDMA test signal and the CCA system. 


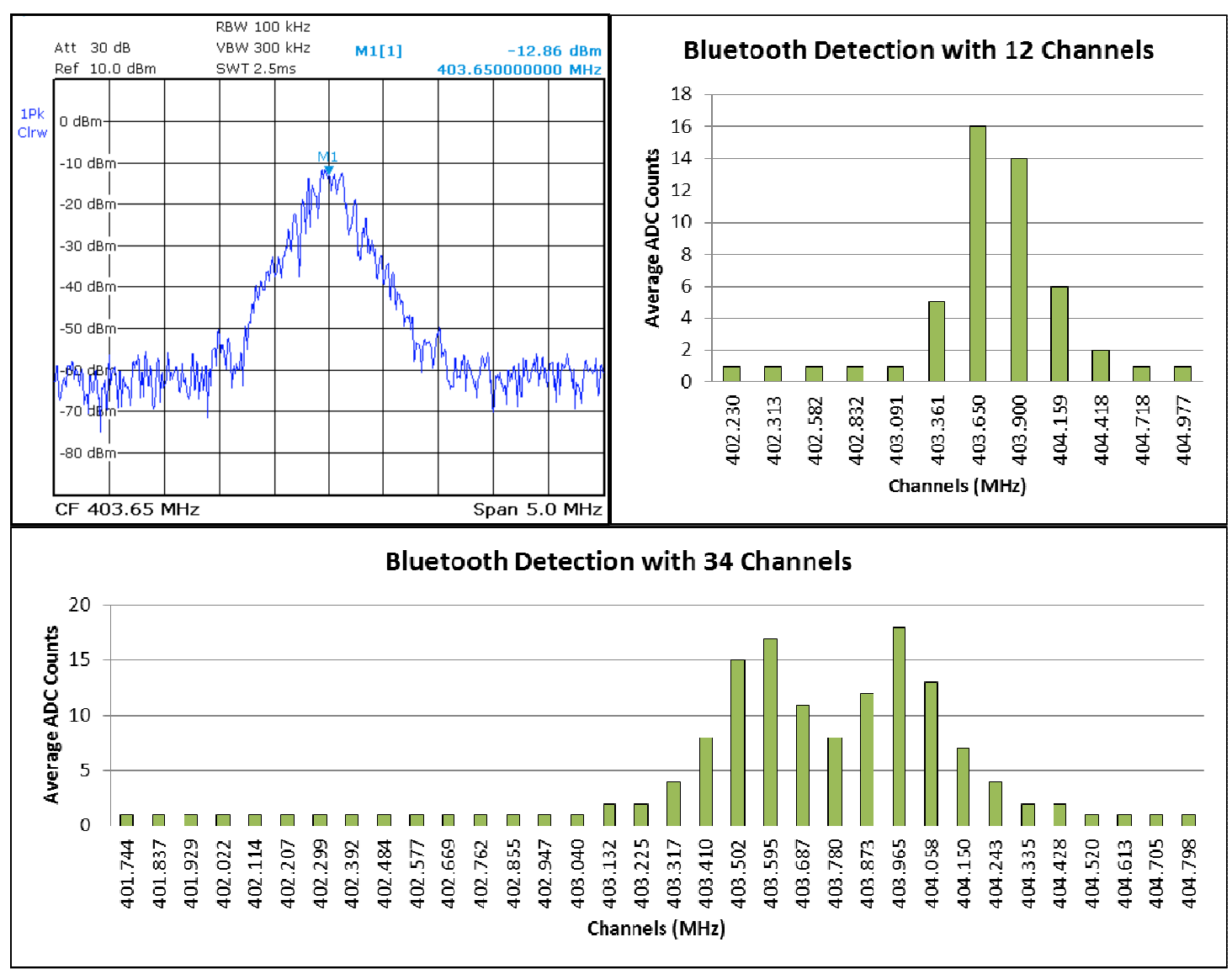

Figure 17 - The Bluetooth modulated test signal and the CCA system output. 


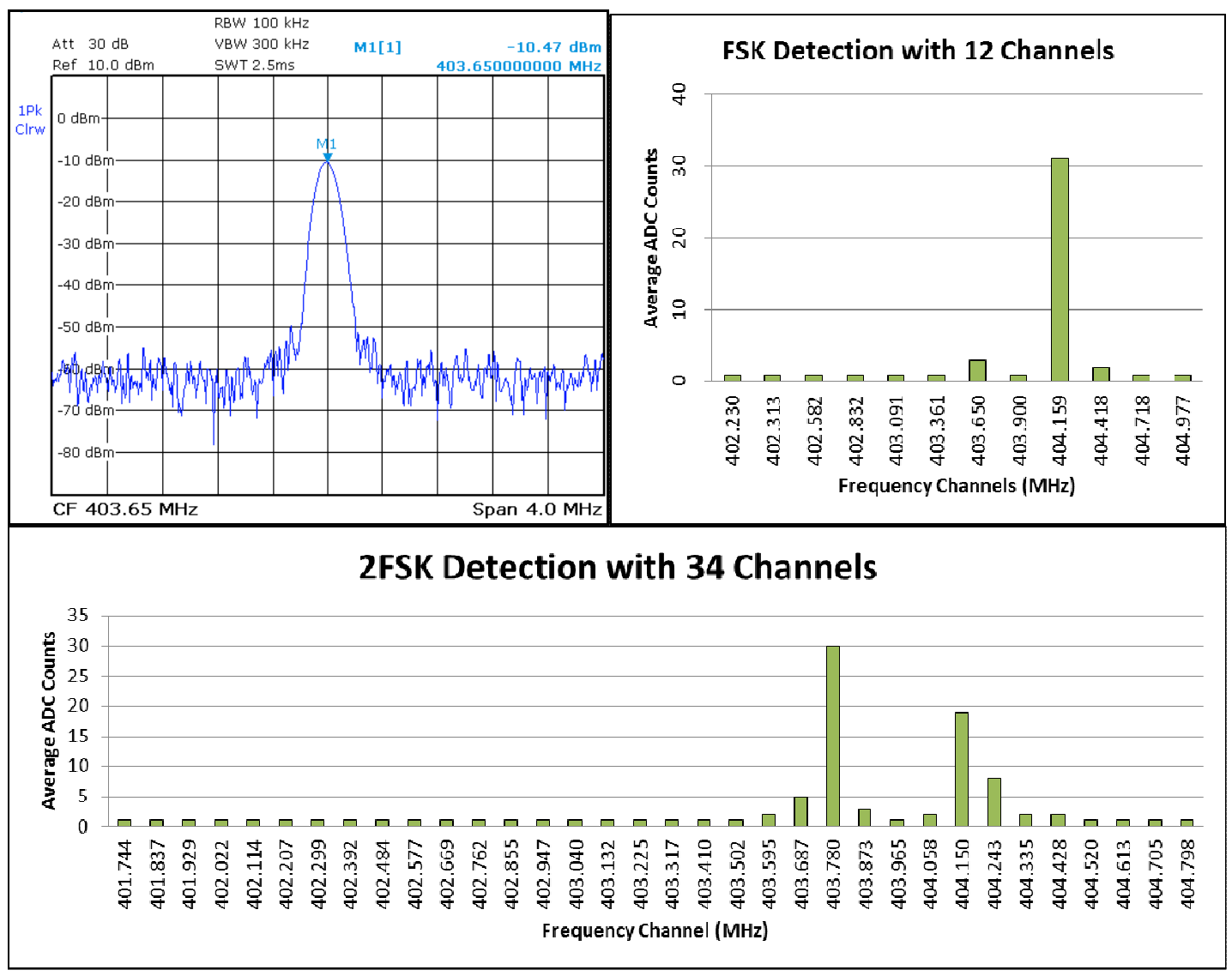

Figure 18 - The 2FSK test signal and the CCA system output.

\subsection{Packet Test Results}

The packet test was intended to show the response of the CCA system to time varying signal levels within the band of interest. The SMIQ controller software shown in Figure 11 was used to configure the SMIQ for the packet test. Each packet consisted of 9 preamble byte and 225 payload bytes including cyclical redundancy check. With Manchester encoding, this gave a packet length of 3744 encoded bits or chips. The CCA controller generated an external trigger pulse with each scan that was used to trigger the SMIQ to send a packet. This provided synchronization of the transmitted packets with the CCA assessment. 
To test the response of the CCA system to both varying packet bandwidth and the duration of packet data activity, the packet content was left fixed and only the data modulation rate was varied. 40 packets were transmitted at each data rate with a center frequency of $403 \mathrm{MHz}$ and a frequency deviation of $70 \mathrm{kHz}$. Energy in a given channel for each packet is considered to be "detected" if there are at least four ADC counts recorded. 


\begin{tabular}{|c|c|c|c|c|c|c|c|c|c|c|c|}
\hline \multirow{2}{*}{\multicolumn{2}{|c|}{ Data Rate (kbps) }} & \multicolumn{5}{|c|}{ Detected Packets } & \multicolumn{5}{|c|}{ Average ADC Counts } \\
\hline & & 8 & 32 & 64 & 256 & 1000 & 8 & 32 & 64 & 256 & 1000 \\
\hline \multicolumn{2}{|c|}{$\mathrm{CBR}(\mathrm{kHz})$} & 156 & 204 & 268 & 652 & 2140 & 156 & 204 & 268 & 652 & 2140 \\
\hline \multicolumn{2}{|c|}{ Active Time (ms) } & 468 & 117 & 58.5 & 14.6 & 3.74 & 468 & 117 & 58.5 & 14.6 & 3.74 \\
\hline \multirow{34}{*}{ 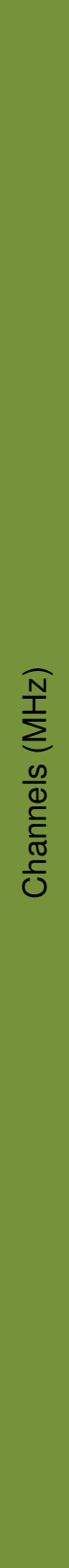 } & 401.744 & 0 & 0 & 0 & 0 & 0 & 2 & 1 & 1 & 1 & 1 \\
\hline & 401.837 & 0 & 0 & 0 & 0 & 40 & 2 & 1 & 1 & 1 & 6 \\
\hline & 401.929 & 0 & 0 & 0 & 0 & 40 & 2 & 1 & 1 & 1 & 22 \\
\hline & 402.022 & 0 & 0 & 0 & 0 & 40 & 2 & 1 & 1 & 1 & 6 \\
\hline & 402.114 & 0 & 0 & 0 & 0 & 40 & 2 & 1 & 1 & 1 & 5 \\
\hline & 402.207 & 0 & 0 & 0 & 0 & 0 & 2 & 1 & 1 & 1 & 1 \\
\hline & 402.299 & 3 & 0 & 0 & 0 & 0 & 2 & 1 & 1 & 1 & 1 \\
\hline & 402.392 & 40 & 36 & 40 & 40 & 0 & 5 & 4 & 5 & 5 & 1 \\
\hline & 402.484 & 0 & 0 & 0 & 0 & 0 & 3 & 2 & 2 & 2 & 1 \\
\hline & 402.577 & 0 & 0 & 0 & 0 & 0 & 3 & 2 & 2 & 2 & 1 \\
\hline & 402.669 & 2 & 0 & 0 & 0 & 0 & 3 & 3 & 2 & 3 & 1 \\
\hline & 402.762 & 40 & 40 & 40 & 40 & 0 & 5 & 7 & 4 & 7 & 1 \\
\hline & 402.855 & 40 & 40 & 40 & 40 & 0 & 20 & 26 & 24 & 24 & 1 \\
\hline & 402.947 & 40 & 40 & 40 & 40 & 0 & 26 & 32 & 30 & 36 & 1 \\
\hline & 403.040 & 40 & 40 & 40 & 40 & 0 & 34 & 33 & 32 & 18 & 1 \\
\hline & 403.132 & 40 & 26 & 40 & 40 & 0 & 7 & 4 & 5 & 5 & 1 \\
\hline & 403.225 & 0 & 40 & 40 & 40 & 0 & 2 & 34 & 9 & 16 & 1 \\
\hline & 403.317 & 0 & 40 & 40 & 40 & 0 & 2 & 28 & 34 & 39 & 1 \\
\hline & 403.410 & 0 & 40 & 40 & 40 & 0 & 2 & 34 & 33 & 25 & 1 \\
\hline & 403.502 & 0 & 40 & 40 & 0 & 0 & 2 & 9 & 13 & 1 & 1 \\
\hline & 403.595 & 0 & 2 & 40 & 0 & 0 & 2 & 3 & 4 & 1 & 1 \\
\hline & 403.687 & 0 & 0 & 0 & 0 & 0 & 2 & 2 & 2 & 1 & 1 \\
\hline & 403.780 & 0 & 0 & 0 & 0 & 0 & 2 & 2 & 2 & 1 & 1 \\
\hline & 403.873 & 0 & 0 & 0 & 0 & 0 & 2 & 1 & 2 & 1 & 1 \\
\hline & 403.965 & 0 & 0 & 0 & 0 & 0 & 2 & 1 & 1 & 1 & 1 \\
\hline & 404.058 & 0 & 0 & 0 & 0 & 0 & 2 & 1 & 1 & 1 & 1 \\
\hline & 404.150 & 0 & 0 & 0 & 0 & 0 & 2 & 1 & 1 & 1 & 1 \\
\hline & 404.243 & 0 & 0 & 0 & 0 & 0 & 2 & 1 & 1 & 1 & 1 \\
\hline & 404.335 & 0 & 0 & 0 & 0 & 0 & 2 & 1 & 1 & 1 & 1 \\
\hline & 404.428 & 0 & 0 & 0 & 0 & 0 & 2 & 1 & 1 & 1 & 1 \\
\hline & 404.520 & 0 & 0 & 0 & 0 & 0 & 2 & 1 & 1 & 1 & 1 \\
\hline & 404.613 & 0 & 0 & 0 & 0 & 0 & 2 & 1 & 1 & 1 & 1 \\
\hline & 404.705 & 0 & 0 & 0 & 0 & 0 & 2 & 1 & 1 & 1 & 1 \\
\hline & 404.798 & 0 & 0 & 0 & 0 & 0 & 2 & 1 & 1 & 1 & 1 \\
\hline
\end{tabular}

Table 3 - CCA packet test performance with 34-channels. 
The 34-channel CCA system output (Table 3) shows the expected results with an increasing number of channels demonstrating energy presence as the bandwidth of the signal increases. At $0.8 \mathrm{~ms}$ per channel, the 34-channel sweep takes 27.2 ms. Packets issued at the $256 \mathrm{kbps}$ and $1000 \mathrm{kbps}$ data rates both conclude before the 34-channel sweep. This is evident in the higher data rate results. For the 12-channel CCA system, a scan takes only $10.4 \mathrm{~ms}$. As shown in Table 4, the 12-channel system is capable of properly detecting the packets at all but the highest data rate tested.

\begin{tabular}{|c|c|c|c|c|c|c|c|c|c|c|c|}
\hline \multirow{2}{*}{\multicolumn{2}{|c|}{ Data Rate (kbps) }} & \multicolumn{5}{|c|}{ Detected Packets } & \multicolumn{5}{|c|}{ Average ADC Counts } \\
\hline & & 8 & 32 & 64 & 256 & 1000 & 8 & 32 & 64 & 256 & 1000 \\
\hline \multicolumn{2}{|c|}{$\mathrm{CBR}(\mathrm{kHz})$} & 156 & 204 & 268 & 652 & 2140 & 156 & 204 & 268 & 652 & 2140 \\
\hline \multicolumn{2}{|c|}{ Active Time (ms) } & 468 & 117 & 58.5 & 14.6 & 3.74 & 468 & 117 & 58.5 & 14.6 & 3.74 \\
\hline \multirow{12}{*}{ 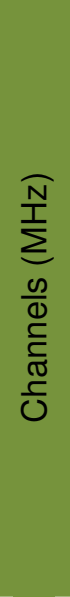 } & 402.023 & 0 & 0 & 0 & 0 & 0 & 2 & 1 & 1 & 1 & 1 \\
\hline & 402.582 & 0 & 0 & 0 & 0 & 40 & 2 & 1 & 1 & 1 & 4 \\
\hline & 402.313 & 0 & 0 & 0 & 0 & 40 & 3 & 2 & 1 & 2 & 7 \\
\hline & 402.582 & 8 & 40 & 40 & 40 & 40 & 3 & 8 & 5 & 7 & 5 \\
\hline & 402.832 & 40 & 40 & 40 & 40 & 40 & 24 & 36 & 32 & 17 & 8 \\
\hline & 403.091 & 40 & 40 & 40 & 40 & 0 & 55 & 33 & 33 & 40 & 1 \\
\hline & 403.361 & 40 & 40 & 40 & 40 & 0 & 6 & 5 & 5 & 5 & 1 \\
\hline & 403.650 & 0 & 40 & 40 & 40 & 0 & 3 & 5 & 5 & 5 & 1 \\
\hline & 403.900 & 0 & 0 & 0 & 0 & 0 & 2 & 1 & 1 & 1 & 1 \\
\hline & 404.159 & 0 & 0 & 0 & 0 & 0 & 2 & 1 & 1 & 1 & 1 \\
\hline & 404.418 & 0 & 0 & 0 & 0 & 0 & 2 & 1 & 1 & 1 & 1 \\
\hline & 404.718 & 0 & 0 & 0 & 0 & 0 & 2 & 1 & 1 & 1 & 1 \\
\hline
\end{tabular}

Table 4 - CCA packet test performance with 12-channels.

5.5. Threshold Detection Vs. Channel Energy Assessment A key point from the packet test results is the tradeoff in using a threshold detection method compared to assessing the signal power channel by channel. Consider the case where all of the channels in a scan returned an ADC count 
value of four or greater. Using the threshold logic, it would appear that the entire band is unusable due to high activity. But if the majority of the channels had energy levels right at the threshold level, there may still be an opportunity to use those channels. This makes selection of the threshold more difficult. Alternatively, having the host controller access the ADC count values directly would make identifying available channels much easier. 
6. Possible Improvements to the CCA System

The CCA system evaluated in this paper is not representative of an ideal CCA system for all use cases. The following discusses some possible improvements that could be made and what realistic performance could be expected.

\subsection{Scan Rate}

In an actual chip radio, the scan rate would be limited by a combination of factors including the frequency synthesizer lock latency and the ADC conversion time. For the ADC used in this work, the conversion time is stated as $1 \mu \mathrm{s}$ and the synthesizer used in the Analog Devices AD7020F-1 transceiver specifies the PLL settling time to be $40 \mu \mathrm{s}$. Summing these components and rounding up to $50 \mu \mathrm{s}$ gives a channel scan rate of $20 \mathrm{kHz}$. For a 25 channel system, a single scan could be conducted in $1.25 \mathrm{~ms}$. This time would be quick enough to catch the preamble of the protocol discussed in section 3.2. These values are approximate but this calculation shows a significant improvement over the CCA system evaluated. Actual scan times would vary greatly depending on the transceiver design.

\subsection{Mixer Type Selection}

As discussed in section 4.1, using an image-reject or SSB mixer would be required to make this design viable. Fortunately most of the transceivers reviewed in this work already have this type of mixer employed. 


\subsection{Multi-Level Reporting or Automatic Threshold Adjustment}

The impacts of directly providing the CCA result register to the host controller is an increase in the transceiver register file size, data transfer time from the transceiver to the controller, and host controller processing time. The host controller may be required to read and process multiple bytes per channel to accurately assess energy contained therein. The threshold method only requires a single bit which cuts down on both storage and processing time requirements but lacks resolution. One alternative to the single threshold may be multiple threshold levels equating to an additional bit per channel in storage and a very slight increase in controller processing time for each threshold level. Two bits per channel could encode four discrete threshold levels. Another alternative would be to have the transceiver monitor the average threshold level across the channels and dynamically increase the threshold to compensate. This option would have a small impact on the transceiver hardware side but would provide improved immunity to situations where the noise level in the band is fairly high.

\subsection{Integration with the Larger Transceiver System} Because a transceiver is a complex system of analog and digital circuitry, it is unlikely that a CCA system can be integrated without additional modification of some transceiver subsystems. In order to perform the channel scan, the receiver gain and filter control mechanisms would need to be activated as well as those systems responsible for frequency synthesis. The process of integrating this feature into a transceiver's hardware architecture will likely uncover many 
common hardware structures while at the same time making the control system more complex. 
7. Conclusion

This research has demonstrated that it is feasible, with a small amount of additional hardware, to augment single chip transceiver designs to include an automated means for frequency channel assessment. This modification is especially well-suited for medical devices which operate in the MedRadio band and are required to monitor in-band activity prior to transmission. The approach may also have application in other bands where channel contention is a concern. With automated channel scanning, medical device manufacturers can shift critical software resources from a task that can easily be done in hardware to focus those resources on beneficial patient therapy and monitoring. 
8. References

1. Federal Communications Commission. (2012, January 27). MedRadio transmitters in the $401-406 \mathrm{MHz}$ band. Code of Federal Regulations Title 4795.6272012 ed.

2. Federal Communications Commission. (2012, January 27). MedRadio transmitters in the 413-419 MHz, 426-432 MHz, 438-444 MHz, and 451$457 \mathrm{MHz}$ bands. Code of Federal Regulations Title 4795.6282012 ed.

3. Cano-García, J.M. An Empirical Evaluation of the Consumption of 802.15.4/ZigBee Sensor Motes in Noisy Environments. 2011 International Conference on Networking, Sensing and Control. Delft, the Netherlands, 11-13 April 2011

4. Ramachandran, I., \& Roy, S. (2007). Clear Channel Assessment in Energy Constrained Wideband Wireless Networks. Wireless Communications, IEEE , vol.14, no.3, June, 70-78.

5. Tekin, A.; Yuce, M.R.; Wentai Liu, "A low power MICS band transceiver architecture for implantable devices," Wireless and Microwave Technology, 2005. WAMICON 2005. The 2005 IEEE Annual Conference, vol., no., pp.55,58, 2005

6. Hsu, Chen-Ming; Lee, Chien-Ming; Yo, Tzong-Chee; Luo, Ching-Hsing, "The low power MICS band biotelemetry architecture and its LNA design for implantable applications," Solid-State Circuits Conference, 2006. ASSCC 2006. IEEE Asian , vol., no., pp.435,438, 13-15 Nov. 2006

7. Cho, Namjun; Bae, Joonsung; Kim, Sunyoung; Yoo, Hoi-Jun, "A 10.8mW body-channel-communication/MICS dual-band transceiver for a unified body-sensor-network controller," Solid-State Circuits Conference - Digest of Technical Papers, 2009. ISSCC 2009. IEEE International, vol., no., pp.424,425,425a, 8-12 Feb. 2009

8. Yang, J.; Fu, M.; Skafidas, E.; Tran, N.; Bai, S.; Mareels, I.; Ng, D.C.; Halpern, M., "A super low power MICS band receiver front-end down converter on $65 \mathrm{~nm}$ CMOS," Biomedical Engineering and Informatics (BMEI), 2010 3rd International Conference on , vol.4, no., pp.1412,1415, 16-18 Oct. 2010 
9. Min, Seungkee; Shashidharan, S.; Stevens, M.; Copani, T.; Kiaei, S.; Bakkaloglu, B.; Chakraborty, S., "A 2mW CMOS MICS-band BFSK transceiver with reconfigurable antenna interface," Radio Frequency Integrated Circuits Symposium (RFIC), 2010 IEEE , vol., no., pp.289,292, 23-25 May 2010

10. Liu, Li-Chen; Ho, Ming-Han; Wu, Chung-Yu, "A medradio-band lowenergy-per-bit CMOS OOK transceiver for implantable medical devices," Biomedical Circuits and Systems Conference (BioCAS), 2011 IEEE , vol., no., pp.153,156, 10-12 Nov. 2011

11. Analog Devices, ADF7020-1 High Performance FSK/ASK Transceiver IC, Datasheet Available Online at: http://www.analog.com/static/importedfiles/data sheets/ADF7020-1.pdf (Accessed on June 23 2012)

12. Atmel, ATA5428 UHF ASK/FSK Transceiver IC, Datasheet Available Online at: http://www.atmel.com/Images/Atmel-4841-Transceiver-ICsATA5428 Datasheet.pdf (Accessed on June 25 2012)

13. Maxim, MAX7030 Low-Cost, $308 \mathrm{MHz}, 315 \mathrm{MHz}$, and 433.92MHz FSK Transceiver with Fractional-N PLL, Datasheet Available Online at: http://datasheets.maximintegrated.com/en/ds/MAX7030.pdf (Accessed on June 26 2012)

14. Maxim, MAX7031 Low-Cost, $315 \mathrm{MHz}, 345 \mathrm{MHz}$, and 433.92MHz ASK Transceiver with Fractional-N PLL, Datasheet Available Online at: http://datasheets.maximintegrated.com/en/ds/MAX7031.pdf (Accessed on June 26 2012)

15. Micrel, MICRF506 410MHz and 450MHz ISM Band Transceiver, Datasheet Available Online at: http://www.micrel.com/ PDF/micrf506.pdf (Accessed on June 23 2012)

16. Nordic, nRF905 Single chip 433/868/915MHz Transceiver, Datasheet Available Online at:

http://www.nordicsemi.com/eng/content/download/2452/29528/file/Product Specification nRF905 v1.5.pdf (Accessed on June 24 2012)

17. NXP, OL2381 Highly Integrated Single-Chip Sub 1 GHz RF Transceiver, Datasheet Available Online at: http://www.nxp.com/documents/data sheet/OL2381.pdf (Accessed on June 25 2012) 
18. RFM, TRC102 400 to $1000 \mathrm{MHz}$ Transceiver, Datasheet Available Online at: http://www.rfm.com/products/data/trc102.pdf (Accessed on June 25 2012)

19. RFM, TRC105 300 to $510 \mathrm{MHz}$ Transceiver, Datasheet Available Online at: http://www.rfm.com/products/data/trc105.pdf (Accessed on June 25 2012)

20. Silicon Labs, Si4430/31/32-B1 ISM Transceiver, Datasheet Available Online at:

http://www.silabs.com/Support\%20Documents/TechnicalDocs/Si4430-3132.pdf (Accessed on June 25 2012)

21. Texas Instruments, CC1120 High Performance RF Transceiver for Narrowband Systems, Datasheet Available Online at: http://www.ti.com/lit/ds/symlink/cc1120.pdf (Accessed on June 23 2012)

22. Texas Instruments, CC1020 Low-Power RF Transceiver for Narrowband Systems, Datasheet Available Online at:

http://www.ti.com/lit/ds/symlink/cc1020.pdf (Accessed on June 23 2012)

23. Texas Instruments, CC1021 Single Chip Low Power RF Transceiver for Narrowband Systems, Datasheet Available Online at: http://www.ti.com/lit/ds/symlink/cc1021.pdf (Accessed on June 23 2012)

24. Kalivas, G. (2009). Digital Radio System Design. Chichester, U.K.: Wiley, Internet Resource.

25. Hu, W.-Y., Lin, J.-W., Tien, K.-C., Hsieh, Y.-H., Chen, C.-L., Tso, H.-T., et al. (2010). A 0.18- m CMOS RF Transceiver With Self-Detection and Calibration Functions for Bluetooth V2.1 EDR Applications. Microwave Theory and Techniques, IEEE Transactions on , vol.58, no.5, May, 13671374.

26. Kleinrock, L., \& Tobagi, F. A. (1975). Packet Switching in Radio Channels: Part I-carrier Sense Multiple-Access Modes and Their Throughput-Delay Characteristics. IEEE Trans. Commun., vol. 23, Dec. , 1400-1416.

27. Stremler, F. G. (1990). 3rd ed. Introduction to Communications Systems . U.S.A.: Addison-Wesley, Print. 
28. Mulagada, R.K.; Yi Yang; Weldon, T.P.; Lieu, D.T., "Single chip spectrum sensing module in CMOS with digital frequency synthesizer and digital output," Southeastcon, 2012 Proceedings of IEEE , vol., no., pp.1,4, 15-18 March 2012

29. Maas, S. A. (1998). The RF and Microwave Circuit Design Cookbook. U.S.A.: Addison-Wesley, Print.

30. Ludwig, R. \& Bretchko, P. (2000). RF Circuit Design Theory and Applications. USA: Prentice Hall, Print.

31. Sayre, C. W. (2008). 2nd ed. Complete Wireless Design. U.S.A.: McGrawHill, Print.

32. Digilent, Nexys3 Board Reference Manual, Available Online at: http://www.digilentinc.com/Products/Detail.cfm?NavPath=2,400,897\&Prod=NE XYS3 (Accessed on July 2, 2012)

33. Texas Instruments, DAC121S101 12-Bit Micro Power, RRO DigitaltoAnalog Converter, Datasheet available online at: http://www.ti.com/lit/ds/symlink/dac121s101.pdf (Accessed on July 2 2012).

34. Analog Devices, AD7991/AD7995/AD7999 4-Channel, 12-/10-/8-Bit ADC with $I^{2} \mathrm{C}$-Compatible Inferface, Datasheet available online at: http://www.analog.com/static/importedfiles/data sheets/AD7991 7995 7999.pdf (Accessed on July 18 2012)

35. Maxim Integrated, MAX2605-MAX2609 45MHz to $650 \mathrm{MHz}$, Integrated IF VCOs with Differential Output, Datasheet available online at: http://datasheets.maximintegrated.com/en/ds/MAX2605-MAX2609.pdf (Accessed on July 6 2012).

36. Avago, MGA-68563 Current-Adjustable, Low Noise Amplifer, Datasheet available online at: http://www.avagotech.com/docs/AV02-0654EN (Accessed on September 2 2012)

37. Minicircuits, TCM4-19+ Surface Mount RF Transformer, Datasheet available online at: http://www.minicircuits.com/pdfs/TCM4-19+.pdf (Accessed on May 22 2012)

38. Skyworks, SMS3626-023 Schottky Diode Quad, Datasheet available online at: 
http://www.skyworksinc.com/uploads/documents/Schottky Quad Mixer Diode s_200040G.pdf (Accessed on July 2 2012) 


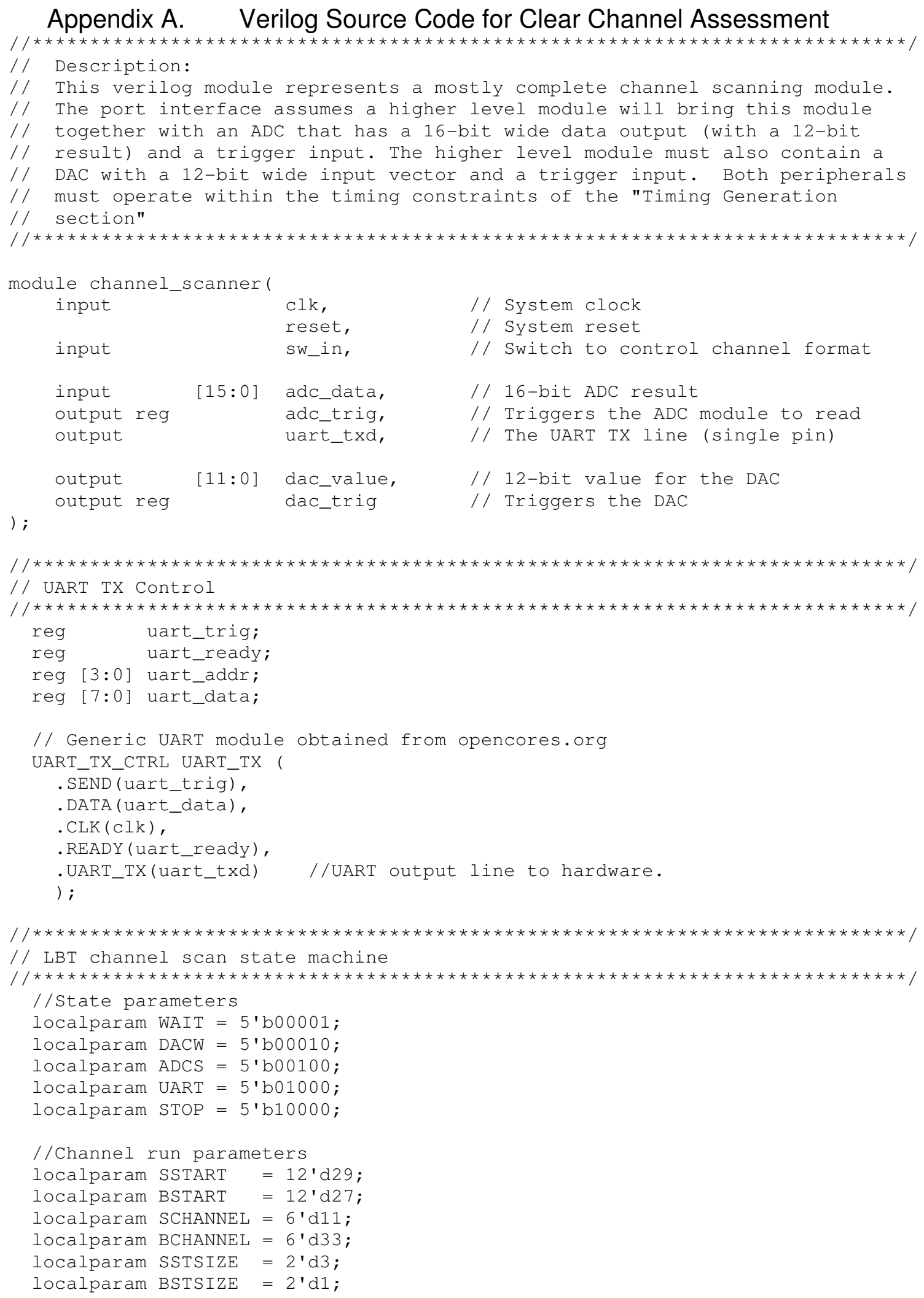




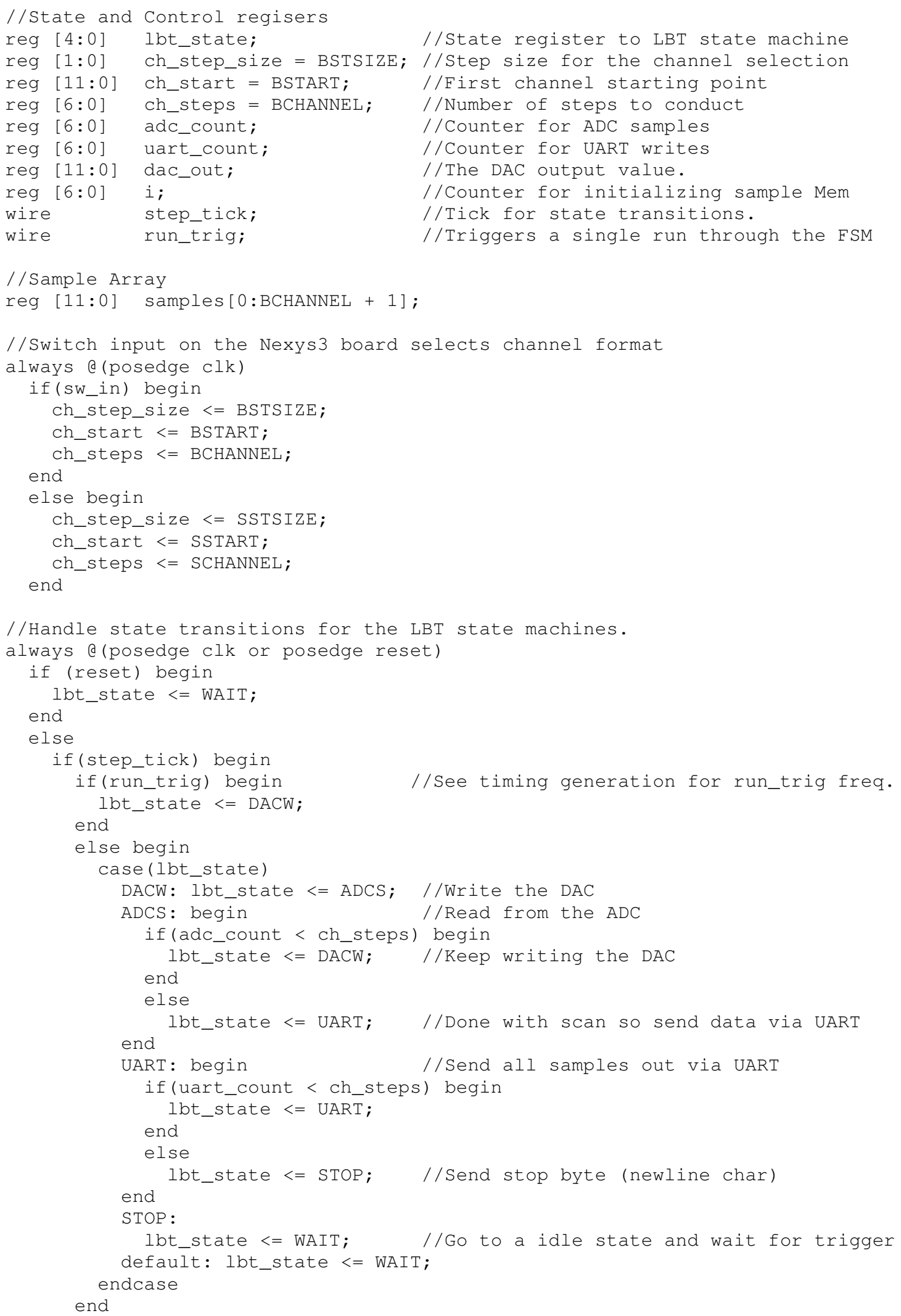




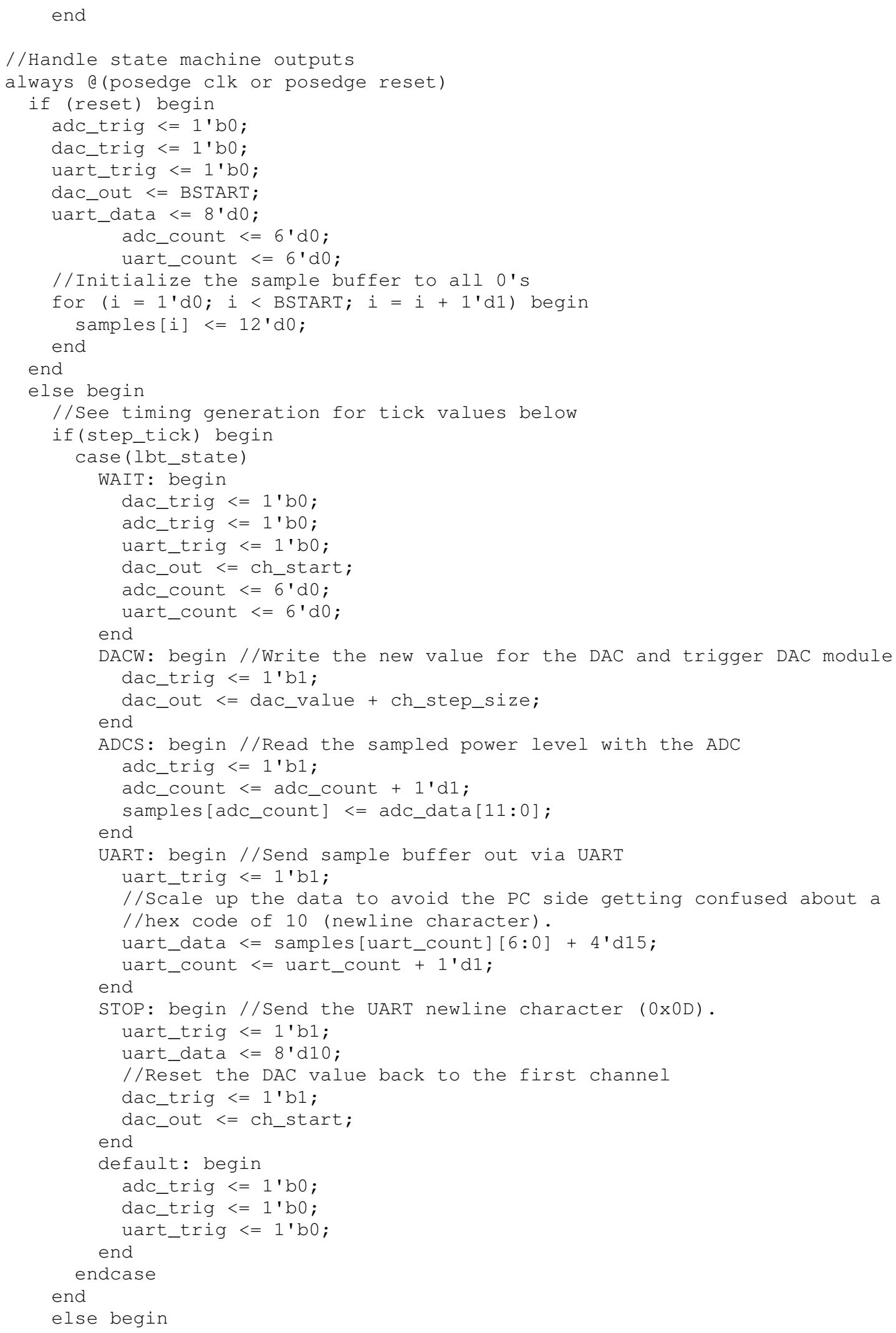




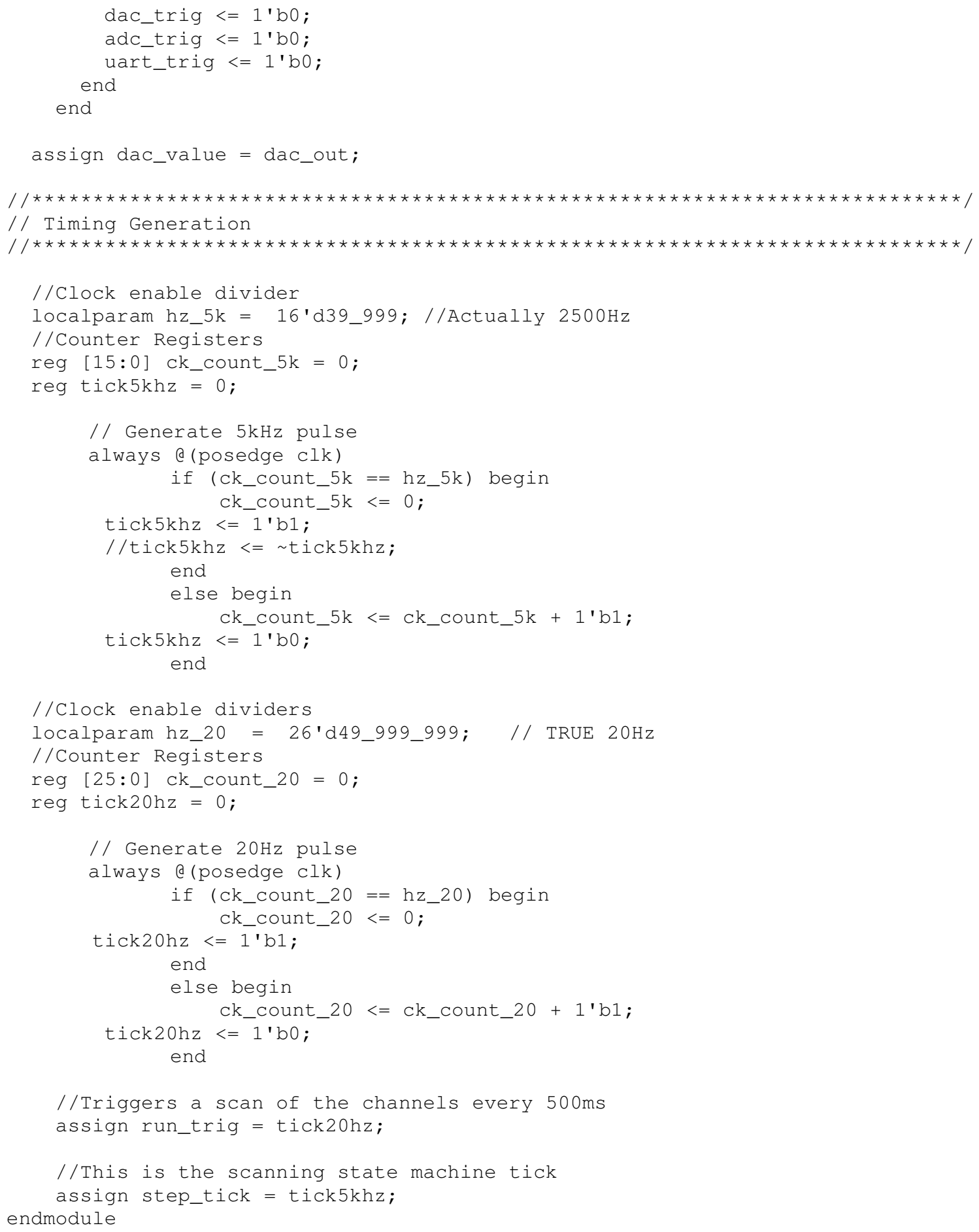


Appendix B. Schematics for RF, DAC, and ADC Hardware

\section{Block Diagram}

Block Diagram:

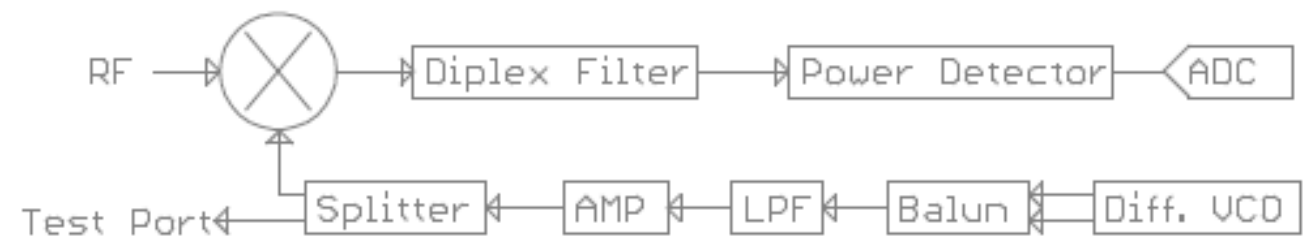

DAC Hardware (Note that only 1 channel was used in this work).

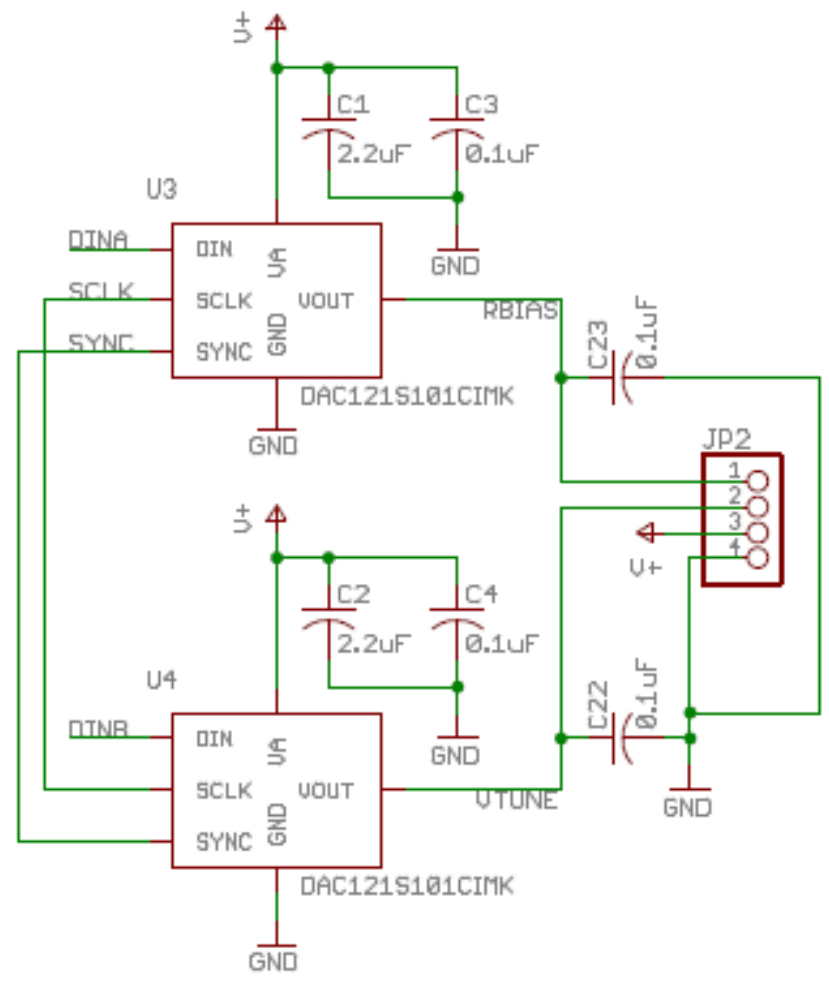

Page $\mathbf{5 4}$ of $\mathbf{5 7}$ 
ADC Hardware (Note only one channel was used in this work

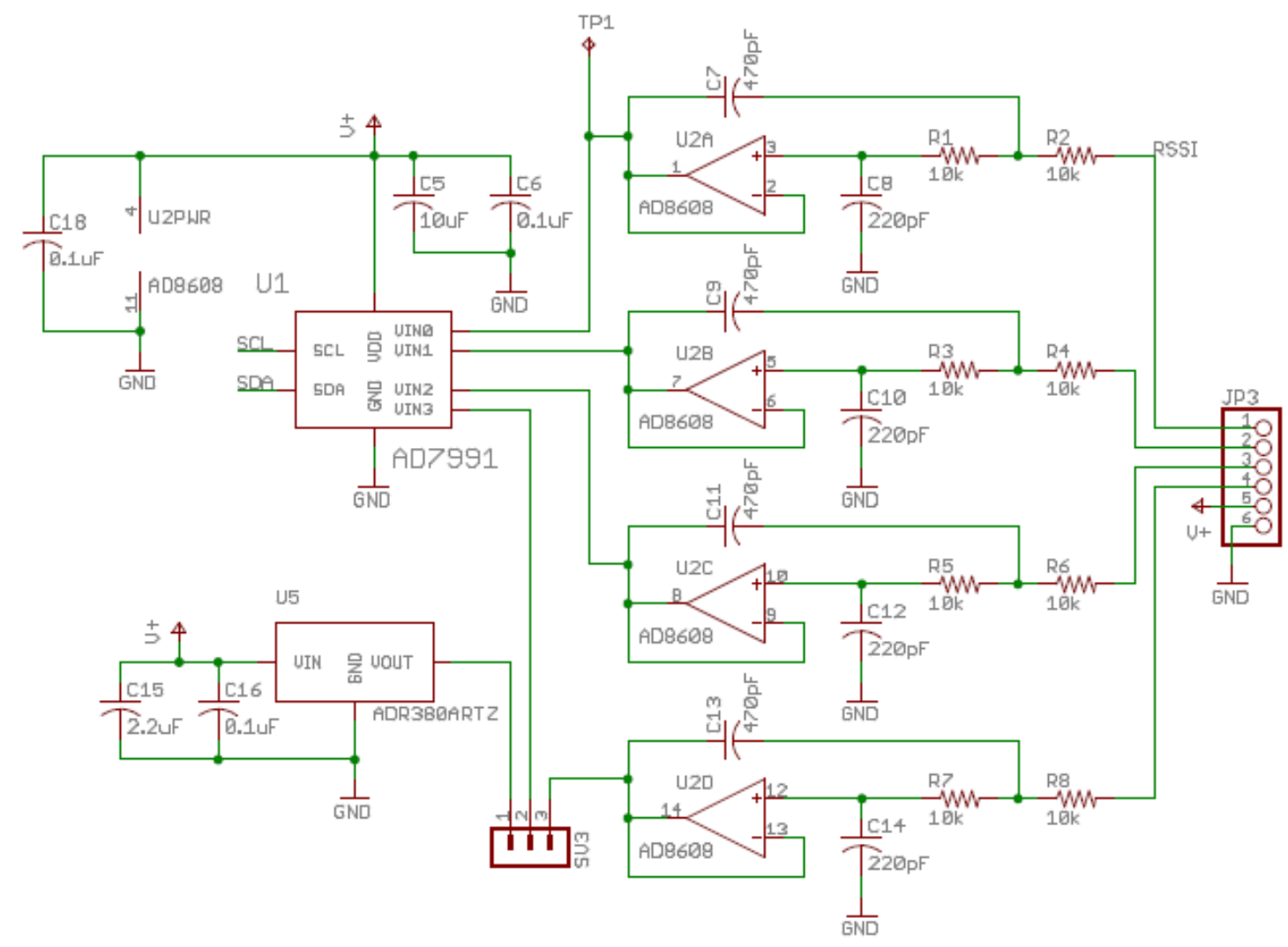

Nexys3 Interface connections

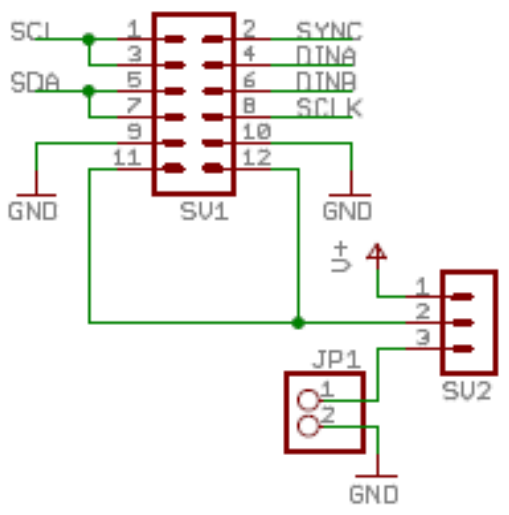

Page 55 of 57 
VCO and amplifier. Note that the MAX2634 amplifier was replaced with the Avago amplifier circuit shown below due to the MAX2634 having too low a compression point.

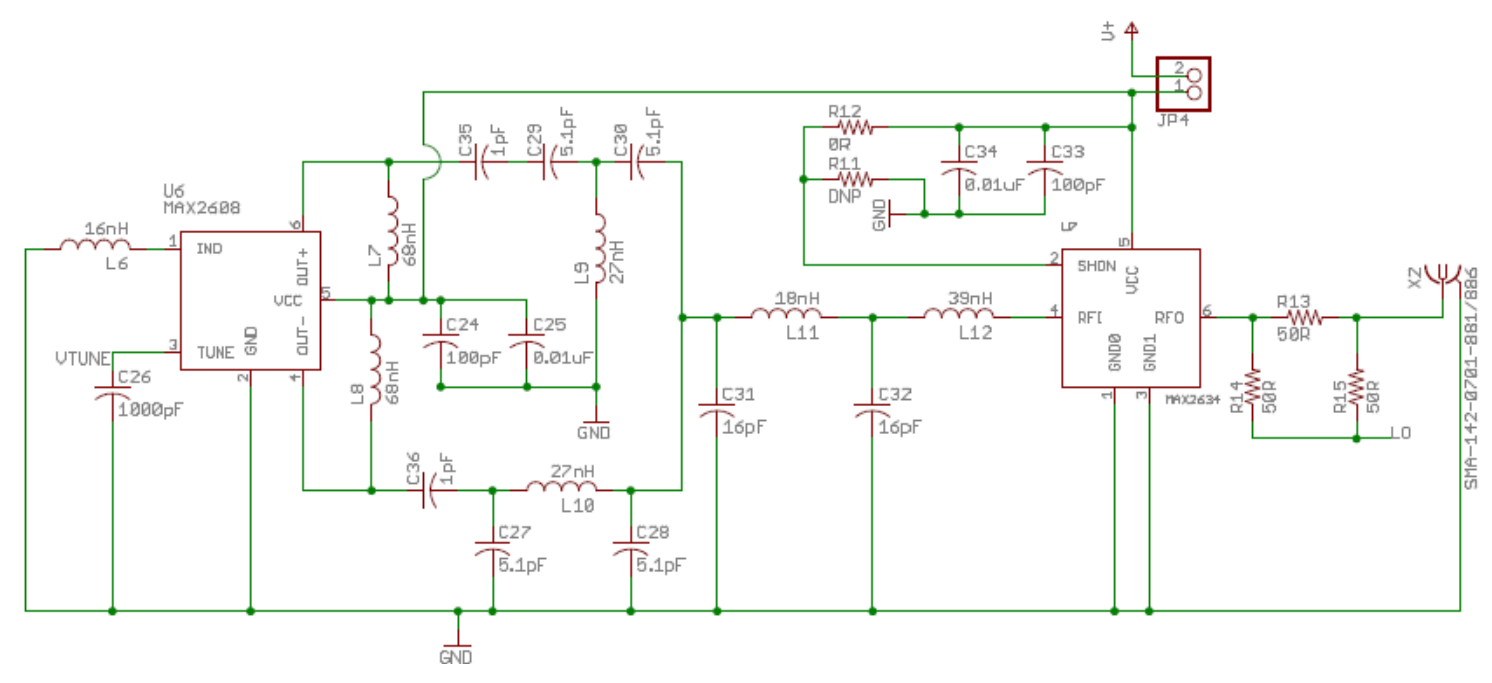

Avago power amplifier for boosting the VCO output to around $+7 \mathrm{dBm}$.

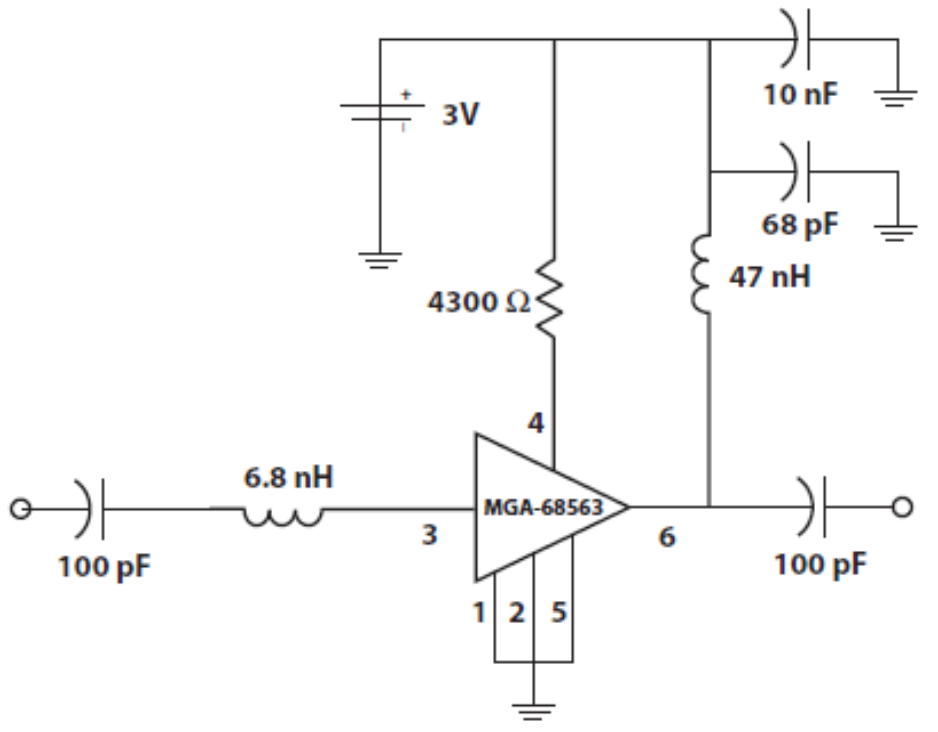

Page $\mathbf{5 6}$ of $\mathbf{5 7}$ 
Mixer, IF diplex filter and Diode detector circuit. Note that the HSMS-2850 was replaced with a Skyworks zero-biased Schottky detector diode.

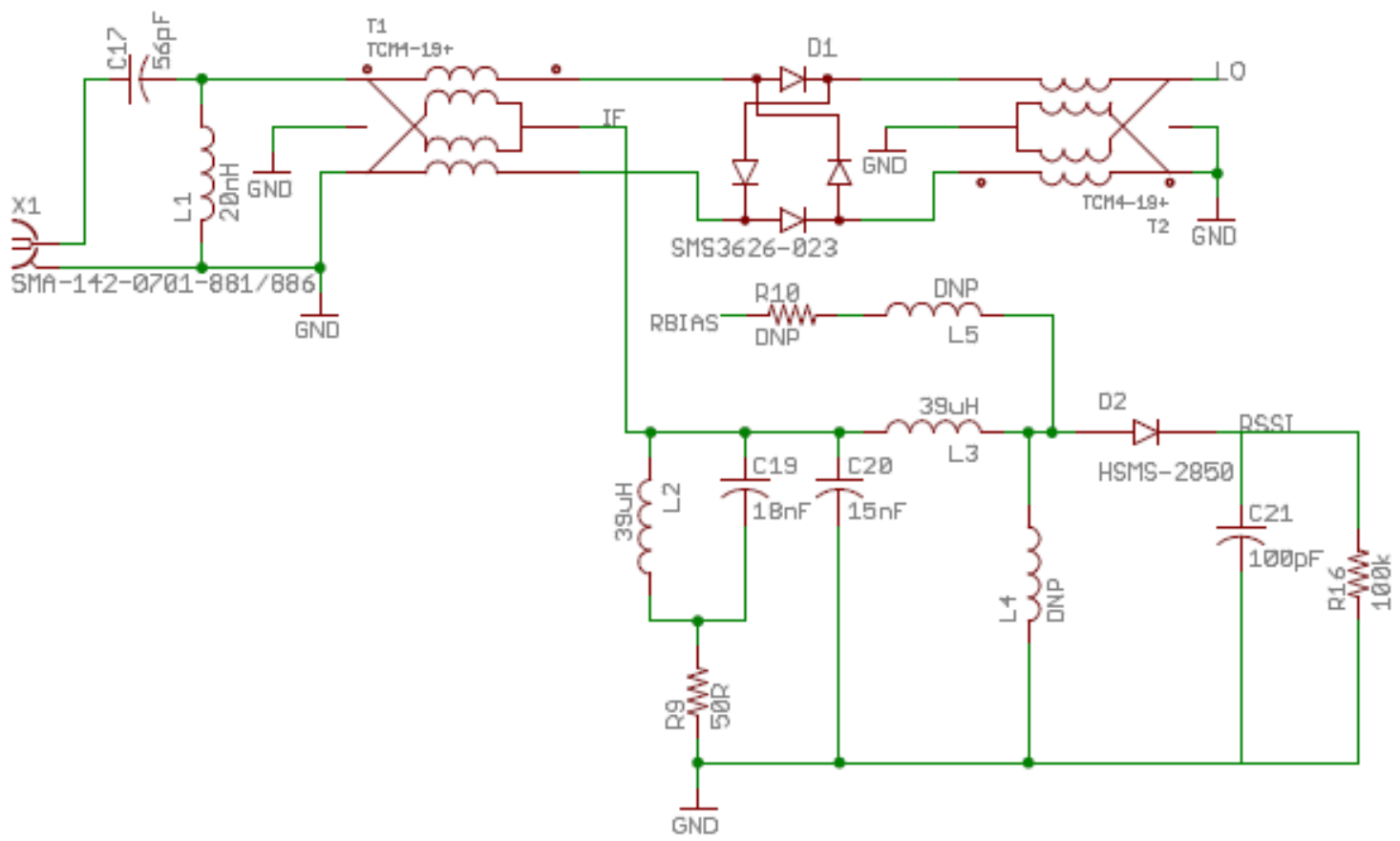

Page 57 of 57 